ARTICLE

DOI: $10.1038 / s 41467-018-06813-x$

\title{
Presenilin-mediated cleavage of APP regulates synaptotagmin-7 and presynaptic plasticity
}

\author{
Gaël Barthet ${ }^{1,2}$, Tomàs Jordà-Siquier ${ }^{1,2}$, Julie Rumi-Masante ${ }^{1,2}$, Fanny Bernadou ${ }^{1,2}$, Ulrike Müller ${ }^{3}$ \&
} Christophe Mulle (i) ${ }^{1,2}$

Mutations of the intramembrane protease presenilin (PS) or of its main substrate, the amyloid precursor protein (APP), cause early-onset form of Alzheimer disease. PS and APP interact with proteins of the neurotransmitter release machinery without identified functional consequences. Here we report that genetic deletion of PS markedly decreases the presynaptic levels of the $\mathrm{Ca}^{2+}$ sensor synaptotagmin-7 (Syt7) leading to impaired synaptic facilitation and replenishment of synaptic vesicles. The regulation of Syt7 expression by PS occurs post-transcriptionally and depends on $\gamma$-secretase proteolytic activity. It requires the substrate APP as revealed by the combined genetic invalidation of APP and PS1, and in particular the APP-Cterminal fragments which interact with Syt7 and accumulate in synaptic terminals under pharmacological or genetic inhibition of $\gamma$-secretase. Thus, we uncover a role of PS in presynaptic mechanisms, through APP cleavage and regulation of Syt7, that highlights aberrant synaptic vesicle processing as a possible new pathway in AD.

\footnotetext{
${ }^{1}$ Interdisciplinary Institute for Neuroscience, CNRS UMR 5297, Bordeaux, France. ${ }^{2}$ University of Bordeaux, F-33000 Bordeaux, France. ${ }^{3}$ Institute for Pharmacy and Molecular Biotechnology, Im Neuenheimer Feld 364, Heidelberg 69120, Germany. Correspondence and requests for materials should be addressed to G.B. (email: gael.barthet@u-bordeaux.fr) or to C.M. (email: christophe.mulle@u-bordeaux.fr)
} 
T he etiology of Alzheimer disease (AD) is complex. The familial forms of Alzheimer disease (FAD) are caused by mutations of two presenilin (PS) paralogs PS1 or PS2 ${ }^{1}$ or of the amyloid precursor protein (APP) ${ }^{2}$ indicating a central role of these genes in the disease. PS is the catalytic subunit of the intramembrane protease $\gamma$-secretase which cleaves several type 1 transmembrane proteins including $\mathrm{APP}^{3}$. The involvement of both PS and APP in the same enzymatic reaction indicates a possible role for APP fragments processed by PS in AD etiology. Within the amyloidogenic pathway, APP is first cleaved by $\beta$ secretase (BACE1) producing the APP $\beta$ Cterminal fragment (APP- $\beta C T F)$, a transmembrane stub further processed through the intramembrane proteolytic activity of $\gamma$-secretase which releases $A \beta$ peptides and the APP-intracellular domain (AICD). While molecular studies have mostly focused on $A \beta$, possible roles for all three APP fragments in AD have been reported ${ }^{4}$. At the clinical level, synaptic dysfunction and loss are the leading pathological correlates of cognitive impairment in $\mathrm{AD}^{5-7}$ suggesting a role for $\mathrm{AD}$-related genes in synaptic transmission.

Accordingly, the roles of both PS and APP in synaptic transmission and plasticity have been studied in several cellular and mouse models (reviewed in ref. ${ }^{8}$ for PS and in ref. ${ }^{9}$ for APP). Both proteins are abundantly expressed in the presynaptic active zone $^{10-12}$, where they interact with proteins of the synaptic release machinery ${ }^{13-15}$. Therefore, the physiological role of both PS and APP in presynaptic mechanisms through interactions with proteins of the presynaptic release machinery needs to be investigated. To address this, we investigated the presynaptic function of PS and APP at hippocampal mossy fiber (Mf) synapses formed between the dentate gyrus (DG) and CA3 pyramidal cells (Mf-CA3 synapses). Mf-CA3 synapses are large synapses with multiple release sites and are characterized by a large dynamic range of presynaptic plasticity ${ }^{16}$. Moreover, Mfs are endowed with a prominent expression of $\gamma$-secretase both in rodents $^{17}$ and in humans ${ }^{18}$. We identified Syt7 as a key presynaptic actor linking the absence of PS or inhibition of $\gamma$ secretase activity to presynaptic accumulation of APP-CTFs and impaired presynaptic mechanisms.

\section{Results}

Combined DG-specific gene deletion with light activation. We have developed optogenetic tools that combine cell-specific activation with cell-specific gene deletion of DG granule cells (GCs) to study the role of PS at Mf-CA3 synapses. The expression of the $\mathrm{C} 1 \mathrm{q} 12$ gene is largely restricted to the DG in the hippocampus ${ }^{19}$. We cloned a sequence of the promoter rich in $\mathrm{CpG}$ islands which includes a $1 \mathrm{~kb}$ fragment upstream of the transcriptional start site and part of the $5^{\prime}$ untranslated region of the mRNA sequence to create a C1q12 minimal promoter (Supplementary Fig. 1a). The minimal promoter was used in a lentiviral bicistronic construct to control the expression of both ChIEF (a variant of ChR2, see ref. ${ }^{20}$ ) fused to the red fluorescent protein tomato and the Cre recombinase, both cDNAs being separated by a $2 \mathrm{~A}$ sequence (Fig. 1a). A similar strategy combining expression of ChIEF and Cre was recently applied using an internal ribosomal entry site (IRES) sequence ${ }^{21}$. In brain sections from mice injected with this construct, ChIEFtom is selectively expressed in the DG (Fig. 1b). Moreover, an extensive study of C1ql2-mediated expression of reporter genes validated the bicistronic $2 \mathrm{~A}$ strategy, the efficiency of the method, the specific targeting of mature Mfs (Supplementary Fig. 1b-e). We used this tool in PS1floxed/PS2KO mice to characterize the properties of Mf-CA3 synapses lacking PS presynaptically, but nonetheless expressing PS1 postsynaptically (Fig. 1c). We confirmed by WB experiments the absence of PS1 protein upon PS1 gene deletion following Cre expression in neuronal cultures from PS1floxed/PS2KO mice (Supplementary Fig. 1f).

We first recorded from ChIEF-expressing GCs in the currentclamp mode, and observed that a $1 \mathrm{~ms}$ light-pulse was sufficient to trigger an action potential (AP) (Supplementary Fig. 1g). Trains of light pulses at $20 \mathrm{~Hz}$ reliably triggered trains of APs up to several seconds without failures (Supplementary Fig. 1g). We did not observe any impact of PS deletion on the excitability of DG GCs and responsiveness to light pulses. At the light intensity used in this study (100\% light intensity, i.e. $2 \mathrm{~mW} / \mathrm{mm}^{2}$ using a $\times 63$ objective and $470 \mathrm{~nm}$ light), GCs fired systematically in both genotypes (Supplementary Fig. 1h). Moreover, the threshold for spike discharge, the amplitude, and the half-width of the AP were similar in both genotypes (Supplementary Fig. 1i).

By illuminating the dendritic layer of the DG, we could activate a restricted population of GCs, and consequently a restricted number of Mfs (Fig. 1b, c and Supplementary Fig. 1j). Mf-EPSCs evoked in CA3 pyramidal cells using this stimulation protocol displayed properties comparable to those of Mf-EPSCs evoked by minimal electrical stimulation ${ }^{22}$. Mean EPSC amplitude, failure rate and short-term plasticity, including paired-pulse facilitation and low-frequency facilitation (at $1 \mathrm{~Hz}$ ) were comparable (Supplementary Fig. 1k) between the two modes of stimulation.

Loss of PS impairs presynaptic facilitation and SV recycling. We virally transduced the C1ql2-ChIEFtom-2A-Cre construct into PS1floxed/PS2-KO mice (PS1 conditional KO mice backcrossed to constitutive PS2 ${ }^{-I}$ mice). This allowed the recording of Mf-EPSCs from presynaptic PSKO Mf-CA3 synapses when the dendrites of Cre-expressing GCs are stimulated by light (Fig. 1c). We compared the properties of EPSCs at WT (ChIEFtom2A-Cre expression in WT mice) and PSKO Mf-CA3 synapses (Fig. 1d). The mean basal amplitude of Mf-EPSCs, their variance and the failure rate were comparable between the two genotypes when GCs were stimulated at low frequency $(0.1 \mathrm{~Hz})$, suggesting similar initial release probability (Fig. 1d, e and Supplementary Fig. 11). To further study basal synaptic transmission, we recorded MfEPSCs in the presence of $32 \mu \mathrm{M}$ of $\mathrm{Cd}^{2+}$ (a bivalent cation that blocks $\mathrm{Ca}^{2+}$ channels), leading to partial inhibition of transmitter release at this concentration (Supplementary Fig. $1 \mathrm{~m}$ ). Under these conditions, $95 \%$ of Mf-EPSCs at $1 \mathrm{~Hz}$ stimulation were estimated to be due to the quantal release of glutamate (see Methods). No difference in the quantal amplitude of Mf-EPSCs between the two genotypes was observed (Fig. 1f, mean: WT $=$ $42.4 \mathrm{pA} ; \quad \mathrm{PSKO}=43.8 \mathrm{pA} /$ median: $\quad \mathrm{WT}=\mathrm{PSKO}=38.0 \mathrm{pA}$ ). Altogether, these results indicate that the absence of PS does not impair basal synaptic properties at low-frequency of stimulation.

We then compared short-term plasticity between the two genotypes. Paired-pulse facilitation ( $40 \mathrm{~ms}$ interval) and frequency facilitation, observed when switching the frequency of stimulation from 0.1 to $1 \mathrm{~Hz}$, did not differ between WT and PSKO Mf-CA3 synapses (Fig. 1g, h). In contrast, the large facilitation triggered by short trains of light stimulation of GC dendrites at $20 \mathrm{~Hz}$ (train facilitation) was markedly smaller at PSKO Mf-CA3 synapses (Fig. 2a). Interestingly, the deletion of presynaptic PS1 alone was sufficient to cause a deficit in train facilitation (Supplementary Fig. 2a). The deficit in train facilitation is unlikely to result from changes in initial release probability as indicated above. Alternatively, PS inactivation could alter the excitability of DG GCs during trains of stimulation. However, we did not observe any difference in spiking activity of GCs in response to trains of light stimulation in GC dendrites. The threshold for spike discharge, the amplitude and the half-width of APs recorded in DG GCs along the $20 \mathrm{~Hz}$ train did not differ between WT and PSKO GCs (Supplementary 
a

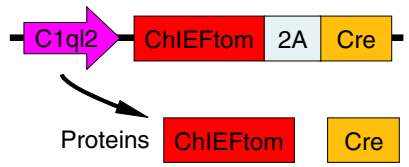

C

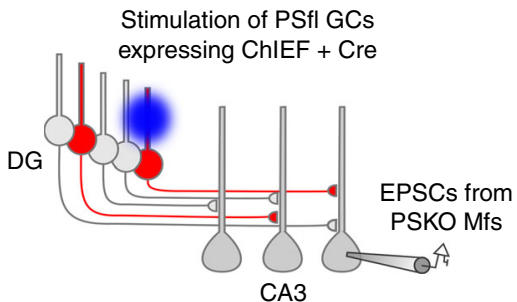

b

\section{GC dendrites}

stimulation

:

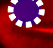

CA3

recordings

d

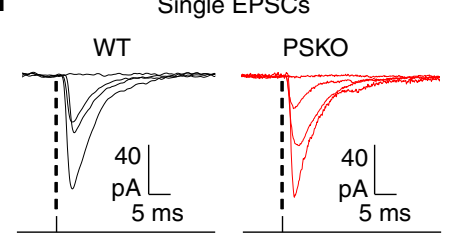

e Amplitude at $0.1 \mathrm{~Hz}$

Failure rate at $0.1 \mathrm{~Hz}$

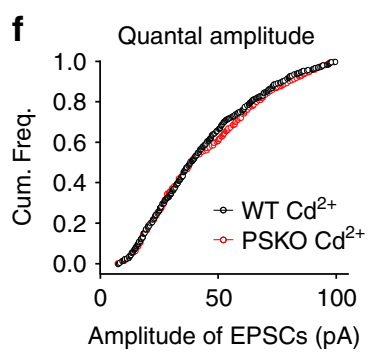

g

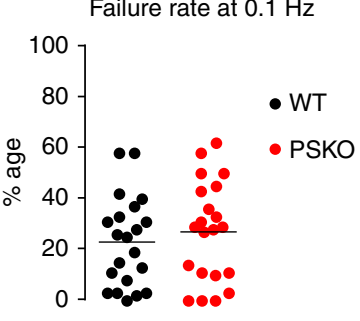
Paired-pulse facilitation

Paired-pulse facilitation
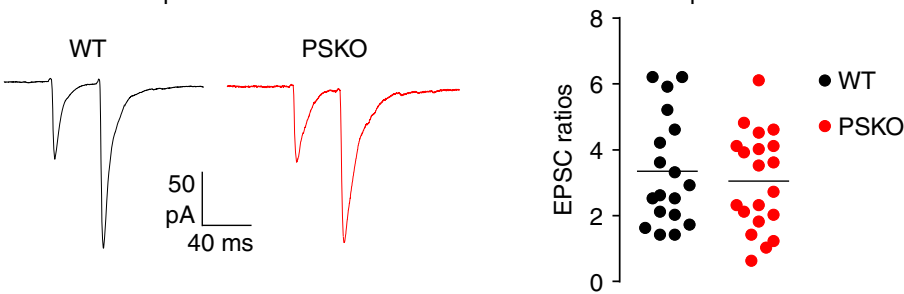

h

Frequency facilitation
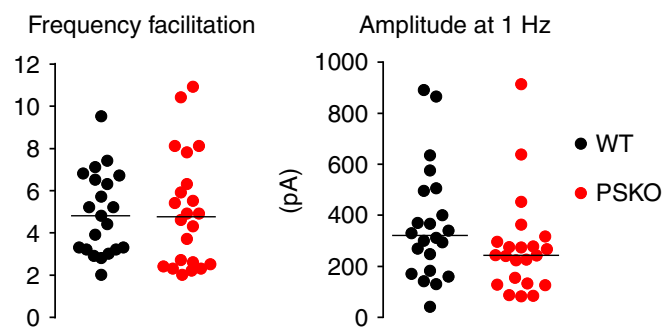

Fig. 1 Study of PS presynaptic function at Mf/CA3 synapses using an optogenetic strategy. a Scheme of lentiviral construct which co-expresses ChIEF fused to the fluorescent protein tomato and the Cre recombinase. The promoter C1ql2 restricts expression to DG granule cells (GCs). b Picture of a parasagittal section of a mouse brain injected with a virus coding for ChIEFtom. c Strategy to selectively study Mf/CA3 synapses that are PSKO presynaptically and intact postsynaptically. The blue light triggers the firing of granule cells that express ChIEF and by obligation (bicistronic construct) the Cre recombinase. In PS "floxed" conditional knock-out mice, the activated GCs do not express PS; EPSCs evoked in CA3 neurons by light pulses in the DG arise from presynaptic PSKO Mf terminals. $\mathbf{d}$ Representative traces of CA3 EPSCs evoked by single light-pulses focused as in (b). e Basal transmission at Mf/CA3 synapses is not altered by PS genotype. Scatter plots with medians of the amplitude, and means of the failure rate of EPSCs recorded in CA3 neurons at $0.1 \mathrm{~Hz}$ in WT or PSKO condition ( $n=22$ /genotype). $\mathbf{f}$ Cumulative frequencies of EPSCs amplitude in presence of $\mathrm{Cd}^{2+}$. $\mathbf{g}$ Representative recordings of paired-pulses EPSCs evoked by two light-pulses separated by $40 \mathrm{~ms}$, and analyses of paired-pulse ratio. The facilitation is represented by the amplitude of the second EPSC normalized to the amplitude of the first EPSC. $\mathbf{h}$ Representative traces of EPSCs evoked by light-pulses at 0.1 or $1 \mathrm{~Hz}$, and analyses of facilitation. The frequency facilitation is represented by the amplitude of EPSCs obtained at $1 \mathrm{~Hz}$ normalized to the amplitude of EPSCs obtained at $0.1 \mathrm{~Hz}$. The raw amplitude of EPSCS at $1 \mathrm{~Hz}$ does not differ between WT and PSKO

Fig. 2b). Overall, inactivation of PS at Mf-CA3 synapses neither affected release probability nor quantal amplitude under basal conditions, but strongly decreased facilitation induced by trains of Mf stimulation by affecting short-term plasticity mechanisms.
The broad dynamic range of EPSC amplitudes from failures $(0.1 \mathrm{~Hz})$ to large currents in response to high-frequency bursts of stimulation represents a high-pass filter for the transfer of information at Mf-CA3 synapses. We explored the impact of the short-term plasticity deficit on spike transfer at DG-CA3 
a

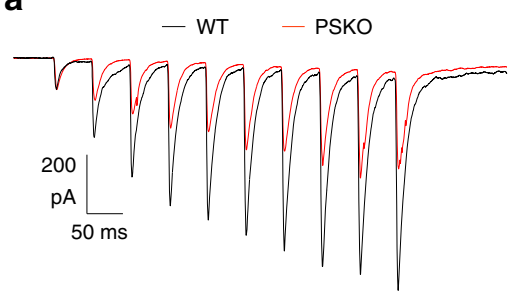

b

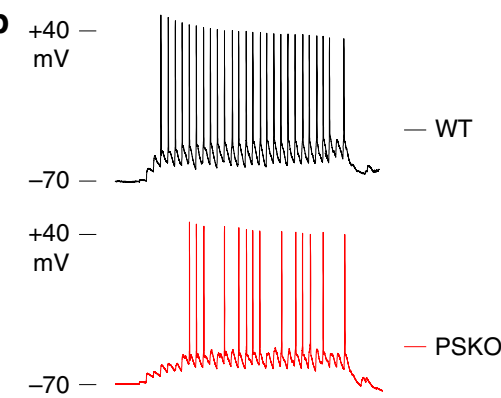

C

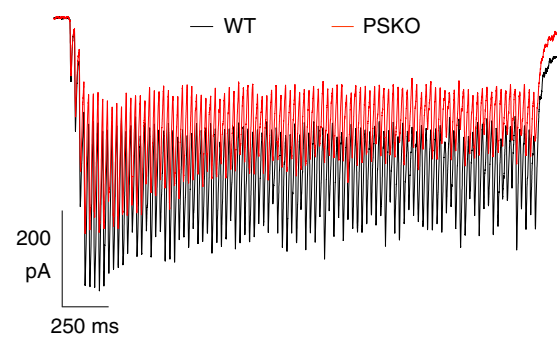

d

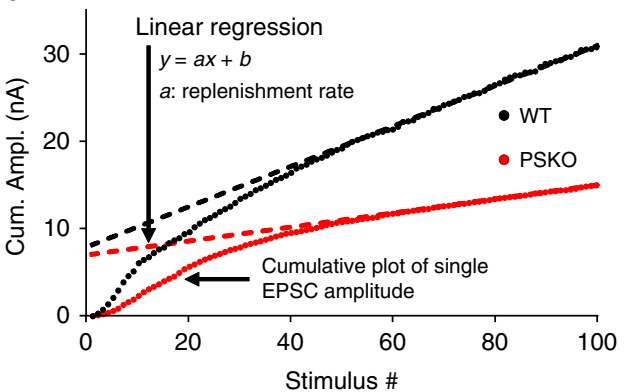

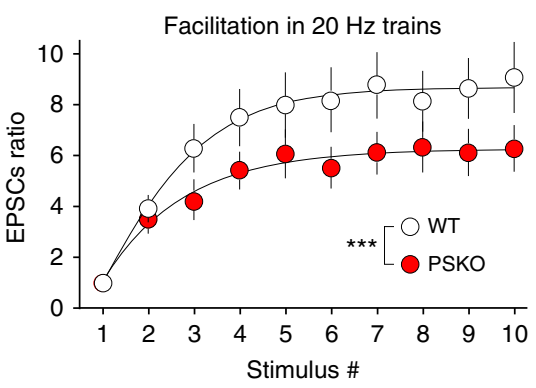

Spike transmission in $20 \mathrm{~Hz}$ trains

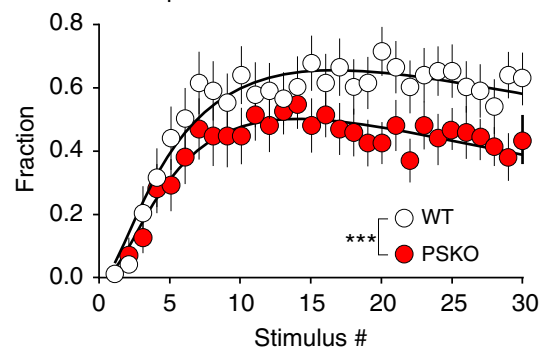

EPSCs amplitude in $40 \mathrm{~Hz}$ trains

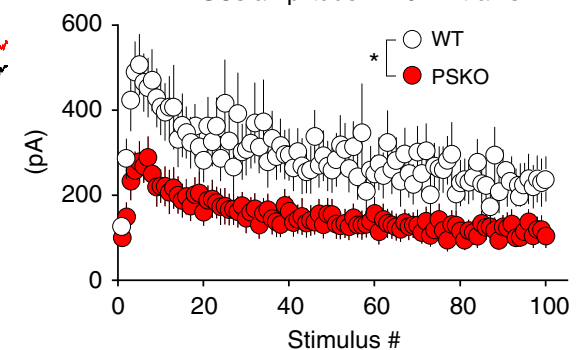

e Replenishment rates

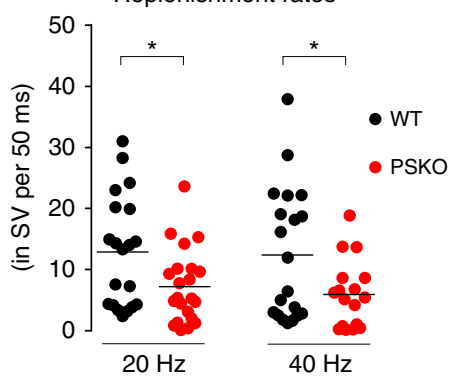

Fig. 2 PS promotes presynaptic facilitation, spike transmission, and SV replenishment. a Representative traces of EPSCs evoked by 10 repetitive light-pulses at $20 \mathrm{~Hz}$, and analysis of the facilitation within the train. The facilitation is represented by the amplitude of EPSCs normalized to the amplitude of the first EPSC. The facilitation is strongly impaired by absence of presynaptic PS. Two-way ANOVA, $n=19$ in WT, 16 in PSKO, $p=0.0004$. b Representative recordings of CA3 spikes in response to 30 stimulations at $20 \mathrm{~Hz}$, and plot of the fractions of spike transmission (for 16 recorded neurons per genotype; five sweeps per neuron) in WT or PSKO genotype. The highest rate of transmission (over 50\%) is reached around 15 stimuli and the plot in PSKO is lower than that in WT condition (two-way ANOVA, $n=16$ in WT, 18 in PSKO $p<0.0001$ ). c Representative EPSCs evoked by 100 stimuli at $40 \mathrm{~Hz}$, and the analysis of their amplitude. After the initial rising phase (facilitation), the amplitude drops and reaches a steady state that is reduced by absence of PS (two-way ANOVA, $n=20$ in WT, 17 in PSKO, $p=0.03$ ). $\mathbf{d}$ Graph of the cumulative amplitude of Mf-EPSCs in WT or PSKO condition from trains of stimulation plotted against the stimulus number. A linear regression fits the second part of the cumulative plot where a steady state is reached. The slope is proportional of the replenishment rate. e Scatter plots with means of replenishment rates calculated neuron per neuron are represented by genotype and frequency. Absence of PS decreases the rates of replenishment (unpaired t-test $p<0.05 ; n=20$ in WT, 22 in PSKO). Errors bars represent s.e.m

connections by performing current-clamp recordings of CA3 pyramidal cells while stimulating GC dendrites with light. In minimal stimulation conditions, the amplitude of Mf-EPSPs at low stimulation frequencies $(0.1-1 \mathrm{~Hz})$ is small and only occasionally triggered spike discharge in CA3 PCs (Supplementary Fig. 2c). Spike transmission increased dramatically in response to trains of stimulation at $20-40 \mathrm{~Hz}$ (Fig. $2 \mathrm{~b}$ and Supplementary 2d) confirming the role of Mf-CA3 synapses as high-pass filters for information transfer within the hippocampal circuit $^{23}$. Within a train of 30 stimuli, the fraction of spike transmission at WT Mf-CA3 synapses increased from 0 to 0.63 after 15 stimuli and then slowly decreased (Fig. 2d). In the 
absence of PS, spike transfer at DG-CA3 connections was markedly less efficient reaching on average a fraction of 0.45 spikes per stimulus after 15 stimuli (Fig. 2b). Altogether, these results indicate that PS is essential for presynaptic facilitation that supports high-pass filtering of spike transfer at DG-CA3 connections.

Synaptic transmission depends on the priming of releasecompetent synaptic vesicles (SVs), a mechanism dynamically regulated by the replenishment of the readily releasable pool of $\mathrm{SVs}^{24}$. Because inactivation of PS impacts Mf-CA3 synapses under conditions of trains of presynaptic stimulation, we postulated that the replenishment of release-competent SVs could be impaired. To test this hypothesis, we used long trains of stimulation at $20-40 \mathrm{~Hz}$ that involve the recycling pool of SVs (Fig. 2c and Supplementary 2e). We estimated the replenishment rate of SVs at Mf-CA3 synapses by plotting the cumulative amplitude of Mf-EPSCs against stimulation number ${ }^{25}$ (Fig. 2d). The rate of replenishment was estimated for each recorded CA3 cell by fitting the linear part of the curve for 100 stimuli trains at $20 \mathrm{~Hz}$ by linear regression (Fig. 2d), and taking into account the average quantal size measured above (Fig. 1f). The replenishment rate at Mf-CA3 synapses was reduced approximately by half in PSKO conditions as compared to WT conditions (WT and PSKO values, from 13 to $7 \mathrm{SVs}$ per $50 \mathrm{~ms}$, Fig. 2e).

Unaltered presynaptic terminals. We investigated whether the reduced facilitation and impaired replenishment rate of SVs resulted from the degeneration of presynaptic Mf terminals lacking PS, possibly leading to smaller Mf boutons with fewer release sites. To investigate this possibility, we studied the morphology of Mf boutons expressing membrane-targeted YFP as a morphological fluorescent marker ${ }^{26}$, by confocal imaging followed by $3 \mathrm{D}$ reconstruction. In both genotypes, Mf boutons had an average volume of $16 \mu \mathrm{m}^{3}$ (on $200 \mathrm{Mf}$ boutons, Supplementary Fig. 2f). We then stained Mf boutons for Bassoon, a presynaptic marker for release sites and we performed stimulated-emissiondepletion (STED) microscopy to evaluate differences in the number of Bassoon puncta between WT and PSKO Mf boutons. We did not observe any difference in the number (16.7 in WT; 17.0 in PSKO) and in the area $\left(0.073 \mu \mathrm{m}^{2}\right.$ in WT; $0.078 \mu \mathrm{m}^{2}$ in PSKO, $n=82$ Mf boutons from three mice per genotype) of Bassoon-positive puncta (Supplementary Fig. 2g) suggesting that impaired facilitation at Mf-CA3 synapses in PSKO does not result from fewer active zones. These morphological data indicate that the impairment of presynaptic function in PSKO is not caused by a broad alteration of the structure or composition of Mf terminals but more likely results from a distinct alteration at the molecular level.

Marked drop of Syt7 axonal expression in the absence of PS. Recent evidence indicates that the $\mathrm{Ca}^{2+}$ sensor synaptotagmin 7 (Syt7) is necessary for both presynaptic facilitation ${ }^{27}$ and replenishment of release-competent $\mathrm{SV}^{28}$, two presynaptic mechanisms being impaired in the absence of PS. We tested whether the functional impairment of PSKO Mf-CA3 synapses results from decreased levels of Syt7 by immunolabelling Syt7 in mouse brain slices. We observed that Syt7 is an abundant protein in the mouse brain (Supplementary Fig. $3 a-c$ ), and is enriched in the stratum lucidum (SL) where Mfs make synaptic contacts with CA3 pyramidal cells, in agreement with its role in synaptic facilitation ${ }^{27}$. Notably, expression of Syt7 in the $S L$ contrasts with the adjacent stratum radiatum (SR) where recurrent CA3/CA3 inputs make synapses devoid of marked presynaptic facilitation (Fig. 3a). In brain slices in which PS is absent only in DG-GCs, the labelling of Syt7 in the $S L$ was dramatically decreased, indicating a loss of Syt7 in PSKO Mf axons (Fig. 3a and Supplementary 3a, d). We measured the mean pixel intensity of staining in the $S L$ and normalized it to the mean pixel intensity in the $S R$, a region not genetically manipulated (Fig. 3b). We observed an average decrease of $40 \%$ (Fig. 3c), a result likely minimized by the infection rate of GCs by the Cre-expressing lentivirus (transduction in around $50 \%$ of GCs). We then prepared protein lysates from laser capture microdissections of the $S L$ and hilus to quantify the expression level of the different Syt7 splice variants. The Syt7 antibody labeled two bands at 46 and $80 \mathrm{kDa}$ (Fig. 3d), corresponding to different splice variants ${ }^{29}$. The shorter variant carries an $\mathrm{N}$-terminal transmembrane region followed by two C-terminal $\mathrm{Ca}^{2+}$-binding domains $(\mathrm{C} 2 \mathrm{~A}$ and $\mathrm{C} 2 \mathrm{~B})^{30}$ and has been shown to rescue presynaptic facilitation in Syt7KO mouse $\mathrm{e}^{27}$. The longer isoform includes extra amino acids of unknown function in the spacer domain localized between the transmembrane region and the $\mathrm{C} 2 \mathrm{~A}$ domain. We observed a dramatic decrease in the expression of the $46 \mathrm{kDa}$ splice variant of Syt7 (Fig. 3d, e) in agreement with decreased staining in brain slices (Fig. 3a and Supplementary Fig. 3a, d).

We then tested whether the functional impairment of SV release at PSKO Mf-CA3 synapses could result from fewer SVs available or from a limited amount of molecules necessary for SV release. To test these possibilities, we labeled brain slices against VAMP2, the most abundant SV protein; against synaptotagmin 1 (Syt1), the $\mathrm{Ca}^{2+}$ sensor necessary for synchronous SV release; against syntaxin1, a SNARE complex protein integrated at the plasma membrane necessary for the release of SV (Fig. 3f); and against Bassoon (Supplementary Fig. 2g). We measured the mean pixel intensity of staining in the $S L$ and did not observe any difference between genotypes for these presynaptic markers (Fig. 3g). Thus, the deficit in protein level observed for Syt7 is not shared by other major proteins of the presynaptic machinery.

The PSKO phenotype is caused by decreased expression of Syt7. We thus postulated that impaired facilitation and SV replenishment at PSKO Mf-CA3 synapses are the functional consequences of decreased Syt7 expression. To address this hypothesis, we tested whether the molecular re-expression of Syt7 in PSKO Mfs was sufficient to recover the functional impairment in synaptic plasticity and SV replenishment rate. We used a strategy involving an injection of PSfl mice with two viral vectors: a first virus allows expression of Cre in GCs leading to deletion of PS and to expression of Cre-dependent ChIEFtom and of Syt7 which is brought by a second virus (Fig. 4a). While GCs infected by only one virus do not respond to light, double-infected GCs lack PS, yet express Syt7 and can be controlled by light. Under this condition, in PSKO, Syt7 was correctly re-expressed in Mf axons (Fig. 4b). The EPSCs evoked by light stimulation in GC dendrites displayed basal synaptic properties similar to WT or to PSKO Mf-CA3 synapses (Supplementary Fig. 4a). However, presynaptic facilitation in response to high frequency trains of stimulation (Fig. 4c, d) and the SV replenishment rate (Fig. 4e, f) recovered to levels not significantly different from WT levels. Besides, the over-expression of Syt7 in WT does not significantly alter presynaptic facilitation or the replenishment rates of SVs (Supplementary Fig. 4b,c). Hence, normal synaptic properties can be rescued in PSKO Mf-CA3 synapses by re-expression of Syt7, demonstrating that the decreased expression of Syt7 is responsible for the PSKO functional phenotype.

$\gamma$-secretase activity regulates Syt7 protein levels. We then asked whether the loss of $\gamma$-secretase proteolytic activity, normally mediated by PS, was involved in the decreased expression of Syt7. We investigated this possibility by treating rat neuronal cultures 
a

WT
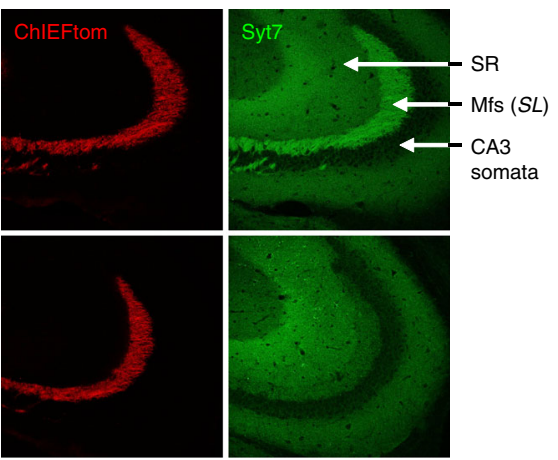

b

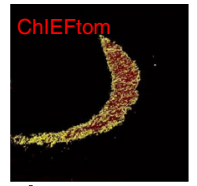

R.O.I.1 (SL) applied to prot. int. channel

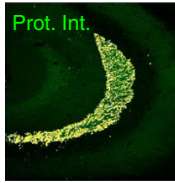

Mean pixel intensity is measured

d

Laser-assisted microdissection of SL
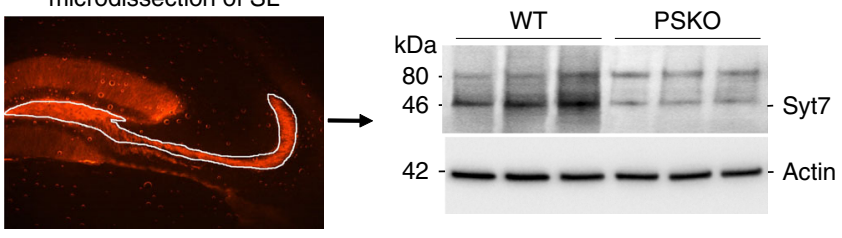

C

Normalization to R.O.I.2 (SR: non-genetically modified region)
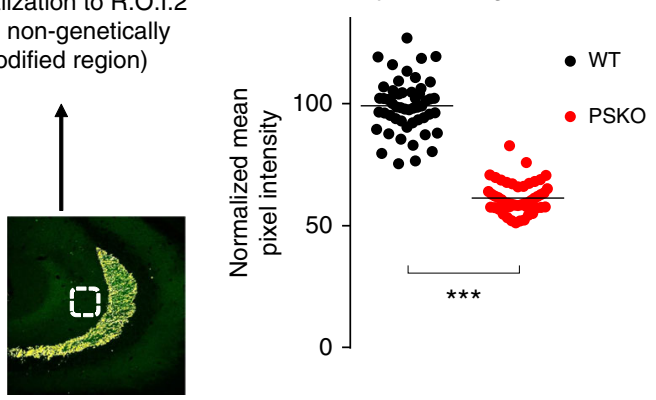

e
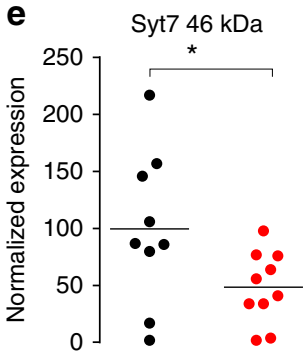

Syt7 $80 \mathrm{kDa}$
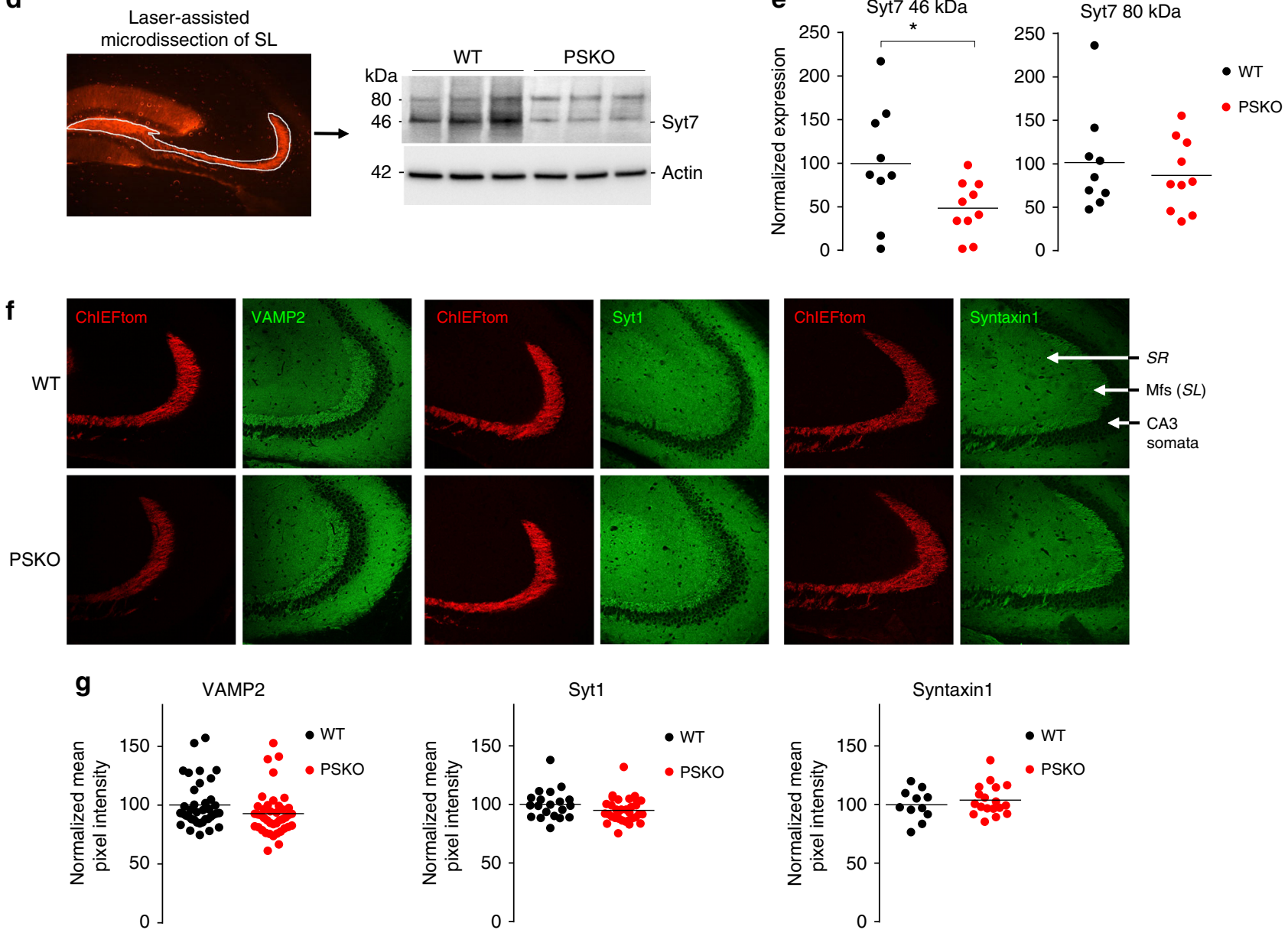

Fig. 3 PS supports the presynaptic expression of Syt7. a Confocal images of the stratum lucidum (SL) where the Mfs can be distinguished in the red channel (ChIEFtom) and stainings of Syt7 depicted in green. Syt7 staining in PSKO Mfs was negligible in comparison to WT. The phenotype presented here using antibody 105173 from Synaptic System is reproduced in Supplementary Fig. S3d with the antibody N275/14 from Millipore. b To quantify the intensity of the stainings a mask corresponding to the $S L$ was created in the red channel. This first region of interest (ROI) was applied to the green channel were the mean pixel intensity of the protein of interest was measured only in the SL. To normalize the results, the mean pixel intensity was also measured in a second ROI: the stratum radiatum (SR). The staining intensities in the $S L$ were normalized to the intensities in the SR. c Scatter plots of the mean pixel intensity in the $S L$ normalized to the mean pixel intensity in the $S R$. A reduction of $40 \%$ of intensity is observed while approximately half of the Mfs are projected from PSKO GCs. $n=53$ slices from nine mice in WT, $n=50$ slices from eight mice in PSKO, $p=0.0004$. d Confocal image of the hippocampus depicting the area dissected with a laser to prepare protein lysates, and western blot from these lysates showing a decreased expression of Syt7. e Quantitative analysis of western blot in c. A $50 \%$ decrease in the expression of the $46 \mathrm{kDa}$ band of Syt7 was detected. f Confocal images of the stratum lucidum (SL) where the Mfs can be distinguished in the red channel (ChIEFtom) and stainings of VAMP2, Syt1 or Syntaxin1A are seen in green. $\mathbf{g}$ Scatter plots of the mean pixel intensity in the $S L$ normalized to the mean pixel intensity in the $S R$ measured in images as in e. Staining patterns and intensities do not differ between genotypes. For VAMP2, $n=38$ slices from eight mice in WT, $n=41$ slices from nine mice in PSKO. For Syt1, $n=20$ slices from five mice in WT, $n=33$ slices from seven mice in PSKO. For Syntaxin1, $n=11$ slices from four mice in WT, $n=18$ slices from seven mice in PSKO 
a

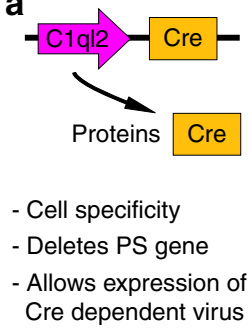

Cre-ON LV based on DIO

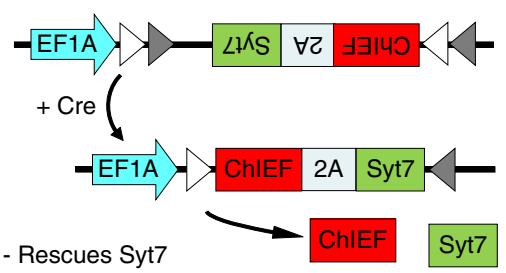

- Allows to control by light the neuronal activity of PSKO neurons rescued for Syt7
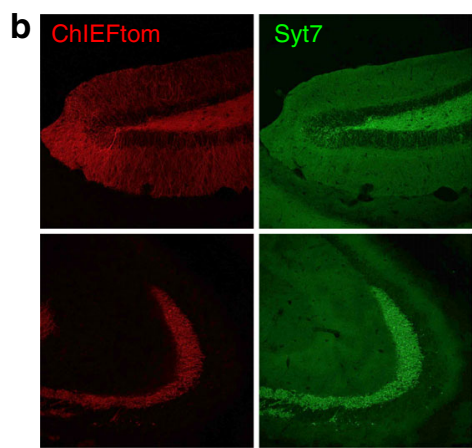

d

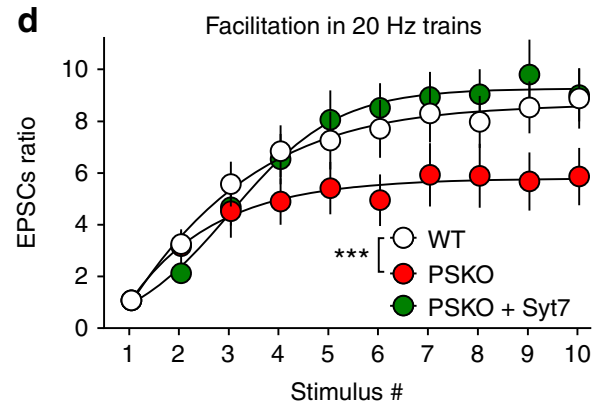

f

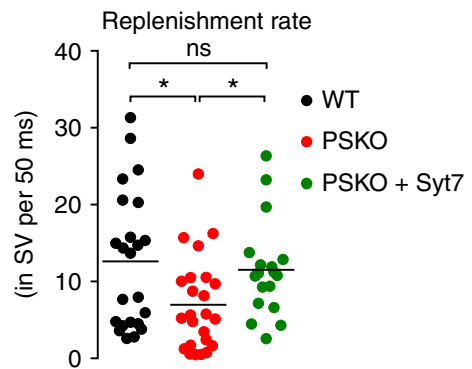

Fig. 4 Impaired facilitation and SV replenishment in PSKO Mfs is caused by decreased Syt7 expression. a Schemes of the two lentiviruses (LV) used to rescue Syt7 expression in PSKO GCs controlled by light. C1ql2 promoter restricts the expression of Cre to GCs where the floxed PS gene is deleted. The Cre also allows the Cre-ON LV to express Syt7 and ChIEF. b Confocal images showing the rescued expression of Syt7 in the hilus (up) and in the stratum lucidum (down) using the strategy described in a. c Representative traces of EPSCs evoked by 10 repetitive light pulses at $20 \mathrm{~Hz}$. $\mathbf{d}$ Analysis of presynaptic facilitation in $20 \mathrm{~Hz}$ trains. Facilitation is represented by the amplitude of the EPSCs normalized to the amplitude of the first EPSC. Facilitation, impaired in absence of presynaptic PS, recovers to WT level by re-expression of Syt7. (Two-way ANOVA, $n=24$ in WT, 23 in PSKO, 24 in PSKO + Syt7, $p=0.001$ ). Errors bars represent s.e.m.) e Representative EPSCs evoked by 100 stimuli at $40 \mathrm{~Hz}$. The amplitude of EPSCs is rescued by Syt7 re-expression. $\mathbf{f}$ Scatter plots with means of replenishment rates calculated per neuron are represented by genotype. The replenishment rate impaired by the absence of PS is rescued by re-expression of Syt7

with a $\gamma$-secretase inhibitor (GSI: L685,458 at $10 \mu \mathrm{M}$ ) for 4 days (from 10 to $14 \mathrm{DIV}$ ) and preparing protein lysates for westernblot (WB) analysis. The Syt7 antibody labelled two bands at 46 and $80 \mathrm{kDa}$ (Fig. 5a), as in the microdissected brain samples. Interestingly, the intensity of the lowest band was decreased by half in conditions of GSI treatment (Fig. 5a, c), indicating that blocking $\gamma$-secretase proteolytic activity downregulates Syt7 expression. In contrast, the expression levels of VAMP2, Syt1, and Syntaxin1 (Fig. 5b, d) were not modified by GSI treatment. Hence, the regulation of Syt7 by $\gamma$-secretase activity is specific among the presynaptic markers tested.

This involvement of $\gamma$-secretase activity indicates a possible role for $\gamma$-secretase substrates expressed at the presynaptic site. APP, the main proteolytic substrate of $\gamma$-secretase is first processed by $\beta$-secretase (BACE1) producing the $\beta$ Cterminal fragment of APP (APP- $\beta$ CTF), a transmembrane stub further cleaved by the intramembrane proteolytic activity of $\gamma$-secretase releasing $A \beta$ peptides and the AICD (Fig. 5e). We hypothesized that the PSKO phenotype, including the decreased expression of Syt7 results from the absence of AICD because this $\gamma$-secretase product has been reported to regulate transcription ${ }^{31}$. We performed laser capture microdissection of the GC layer to prepare RNA samples used for qRT-PCR of Syt7 mRNA, and did not observe any change between the two genotypes (Fig. 5f). We further addressed the possible involvement of AICD in presynaptic mechanisms by testing whether the molecular rescue of AICD in PSKO GCs could recover the functional phenotype. Once again we used a strategy based on the combination of a Creexpressing lentivirus with a Cre-ON lentivirus (Fig. $5 \mathrm{~g}$ and Supplementary 5a). This allowed the optical control of activity of GCs which were PSKO but still expressed AICD (Fig. 5h). In this condition, we did not observe any recovery of presynaptic facilitation (Fig. 5i) while basal transmission was not affected (Supplementary Fig. 5b,c). The lack of evidence for an impairment at the transcriptional level indicates that Syt7 protein is dysregulated post-transcriptionally.

Syt7 downregulation results from accumulation of APP-CTFs. In the absence of PS and its $\gamma$-secretase proteolytic activity, unprocessed substrates APP-CTF $/ \beta$ (APP-CTFs) may 
a

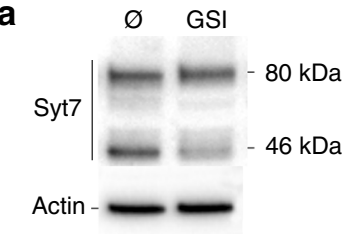

b

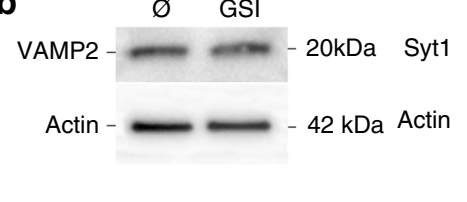

$\varnothing \quad$ GSI

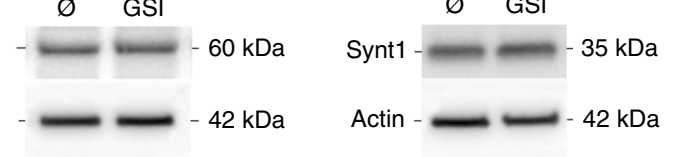

C $46 \mathrm{kDa}$ Syt7 expression

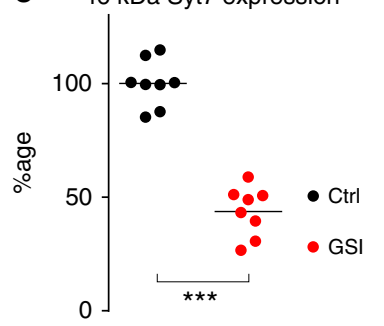

d

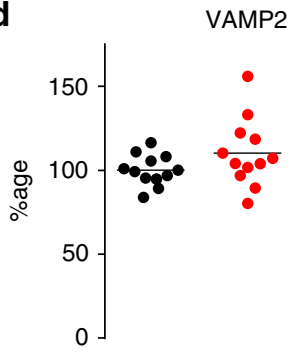

Syt1

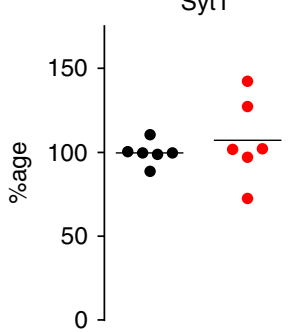

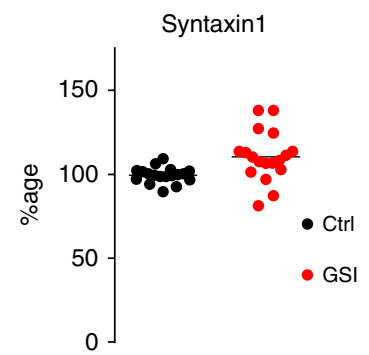

e APP proteolytic metabolism
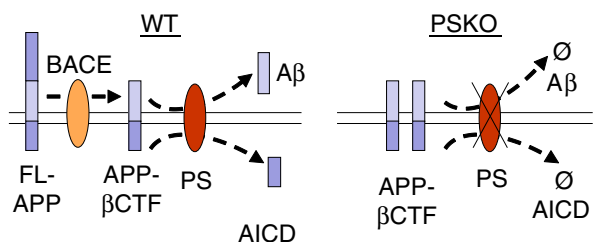

$\mathbf{f}$

Laser-assisted microdissection of GCs

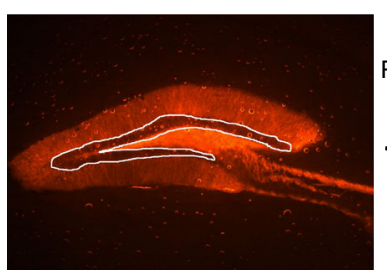

RT-qPCR

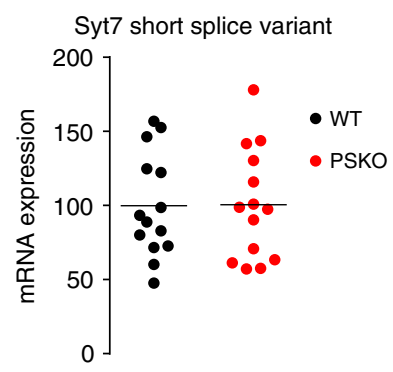

g

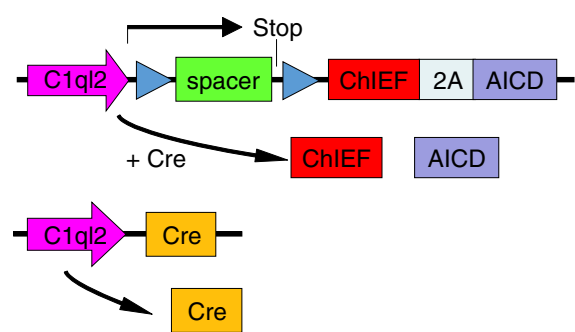

h

Stimulation of PS-KO neurons
expressing ChIEF, Cre and AICD

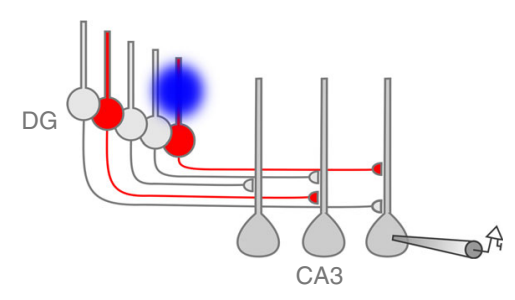

i

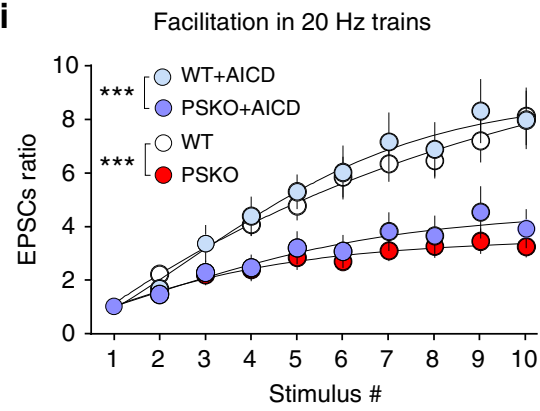

Fig. $5 \gamma$-secretase activity regulates Syt7 protein levels. a, b Western blots of protein samples from neuronal cultures treated or not with $10 \mu \mathrm{M}$ GSI from 10 to 14DIV. The $46 \mathrm{kDa}$ Syt7 band decreases dramatically when $\gamma$-secretase is inhibited. $\gamma$-secretase inhibition does not impair expression of VAMP2, Syt1, and Syntaxin1A. c, d Scatter plots of the western-blot quantification of samples from neuronal culture treated or not with GSI. Syt7 expression is decreased by half in treated condition as seen in $\mathbf{a}$, while the expression of VAMP2, Syt1, and Syntaxin1A is stable under GSI condition as seen in $\mathbf{b}$. e Schemes of APP proteolytic metabolism in WT and PSKO conditions. Full-length (FL) APP is first cleaved by BACE producing $\beta C T F$, a transmembrane stub, further degraded by PS ( $\gamma$-secretase) into A $\beta$ and APP IntraCellular Domain (AICD). In absence of PS, $\beta C T F$ cannot be catabolized and thus accumulates. $\mathbf{f}$ Confocal image of the hippocampus depicting the GCs layer dissected with a laser to prepare RNA samples, and RT-qPCR analysis of the samples indicates that mRNA level of Syt7 is not impaired in absence of PS. $\mathbf{g}$ Schemes of the two lentiviruses (LV) used to rescue AICD expression in PSKO GCs controlled by light. C1q12 promoter restricts the expression of Cre to GCs where the PS floxed gene is deleted. The Cre also removes the Lox-Stop-Lox sequence allowing the CreON LV to express AICD and ChIEF. h Strategy to selectively study Mf/CA3 synapses that are presynaptically PSKO, yet express AICD. In PS "floxed" conditional knock-out mice, double-infected GCs do not express PS (Cre deletes PSfl gene), yet express AICD (Cre dependently), and ChIEF (bicistronic construct) allowing the control of their activity by light. GCs infected by a single virus do not respond to light. i Facilitation of EPSCs amplitude measured along trains of 10 stimuli at $20 \mathrm{~Hz}$ in WT or PSKO-expressing AICD. The impaired facilitation of PSKO is not rescued by expression of AICD. Two-way ANOVA, $n=8$ in WT, 11 in PSKO, $p=0.017$. Errors bars represent s.e.m

accumulate. We studied the expression level of different fragments of APP by staining brain slices with antibodies directed against either the Nterminal or Cterminal epitopes of APP. In control conditions, we observed that the $S L$ was moderately stained with the Cterminal antibody relatively to the CA3 somata and barely stained with the Nterminal antibody (Fig. 6a). Interestingly, in conditions where PS is selectively suppressed in Mf axons, we observed a striking increase of APP staining with the
Cterminal but not with the Nterminal antibody indicating that an APP fragment lacking the Nterminal epitope accumulates in PSKO axons (Fig. 6a, b). Using protein samples obtained by laser microdissection of PSKO Mfs, we distinctly identified APP-CTFs as the APP fragment accumulating in Mf axons (Fig. 6c, d). In Mfs, the initial ectodomain shedding of APP is likely performed by BACE1 which is abundantly expressed in these axons (ref. ${ }^{32}$ and Supplementary 6a). In PSKO Mfs, the accumulation 

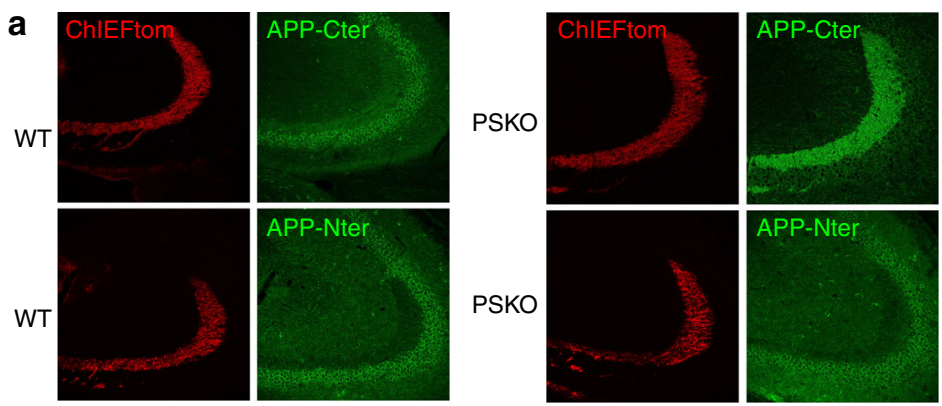

C Samples from microdissected SL

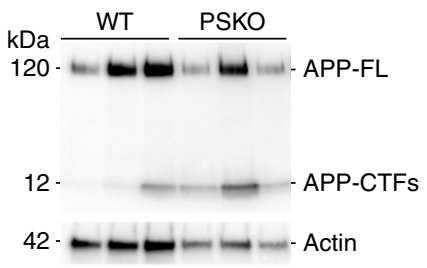

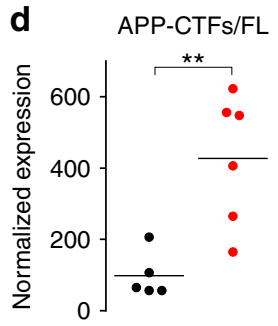

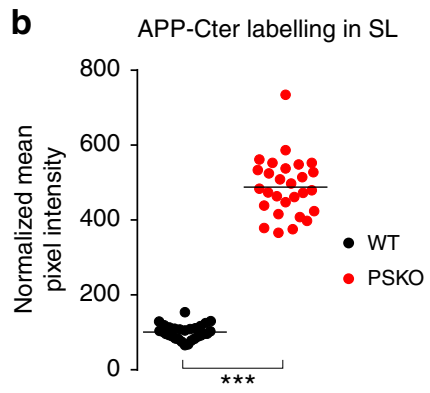

$\mathbf{e}$

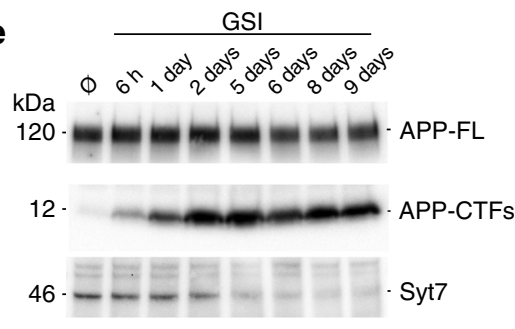

Fig. $6 \gamma$-secretase proteolytic activity regulates the axonal expression of APP- $\beta$ CTF. a Confocal images of the Mfs tracts distinguished in the red channel (ChIEFtom) and of stainings of APP Cterminal with Y188 antibody (upper panel) and Nterminal with JRD32 antibody (lower panel). In WT, Mfs are labelled with the Cterminal but barely with the Nterminal antibody. In PSKO, Mfs are strongly labelled with the Cterminal but not with the Nterminal antibody. b Scatter plots of the mean pixel intensity of APP-Cter in the SL normalized to the mean pixel intensity in the SR. A five-time increase of the intensity is observed in PSKO condition compared to WT. c Samples prepared as in (Fig. 3c) show a striking increase in APP-CTFs levels in dissected Mfs detected with Y188 antibody against APP-Cter. d Quantitative analysis of western blot in c. A 400\% increase in the expression of APP-CTFs was detected. e Western-blot of samples prepared from neuronal cultures treated with GSI from 6 h to 9 days in vitro. APP-CTFs accumulate while Syt7 drops

of APP- $\beta$ CTF in terminals suggests that it can neither be retrogradely transported nor be catabolized by enzymes other than PS. We induced an accumulation of APP-CTFs in neuronal cultures similar to the one observed in Mfs by blocking PS function with a $\gamma$-secretase inhibitor (GSI). Interestingly, we observed an inverse correlation between Syt7 expression level and APP-CTFs, which progressively accumulates in protein lysates from cultures treated from $6 \mathrm{~h}$ to 9 days with GSI (Fig. 6e), suggesting that accumulation of APP-CTFs prevents the normal expression of Syt7.

Amongst other $\gamma$-secretase substrates identified so far only a few of them are known to be expressed at the presynaptic site and could potentially play a role in the presynaptic phenotype observed $^{33,34}$. We identified $\mathrm{N}$-cadherin and Neurexin1 as additional candidate substrates that may accumulate in presynaptic Mf terminals upon deletion of PS. We studied the accumulation of the transmembrane stubs of these proteins produced after ectodomain shedding in neuronal cultures treated with GSI (Supplementary Fig. 6b-e). We also stained hippocampal slices using antibodies against the Cterminal fragments of these two proteins. We observed, as already reported, that NcadCTF1 accumulates in neuronal cultures ${ }^{35}$ but not in PSKO Mf terminals. The Cterminal stub of Neurexin does not accumulate in cultured neurons treated with GSI nor in PSKO Mfs (Supplementary Fig. 6b-e).

To address whether there was a causal relation between APPCTFs accumulation and Syt7 downregulation, we crossed the PS1fl mouse line with an APPfl mouse line ${ }^{36}$. In the resulting APPfl/PS1fl mouse line, the expression of Cre removes both PS1 and APP in the same cells; thus even though the enzyme is removed, no substrate (APP-CTFs) can accumulate (Fig. 7a). We compared the expression level of Syt7 in APPfl/PS1fl to PS1fl and WT (Fig. 7b, c). In PS1KO Mfs from PS1fl mice, the level of Syt7 expression was significantly decreased compared to WT. However, the extent of this reduction was less pronounced than the reduction observed in PSKO Mfs from PS1fl/PS2KO mouse. This is in agreement with the milder impairment of presynaptic facilitation observed in PS1KO compared to PSKO (removal of both PS1 and PS2) conditions (Supplementary Fig. 2a). In Mfs expressing neither PS1 nor APP, the expression level of Syt7 was comparable to WT. Thus, the absence of APP prevents the decreased expression of Syt7 in Mfs, demonstrating a causal relationship between APP processing and downregulation of Syt7. While the level of Syt7 differs between PSKO and APPKO/ PS1KO conditions, $\gamma$-secretase products of APP processing (A $\beta$, AICD) cannot be produced in both conditions, indicating that the Syt7 deficit does not result from the absence of $A \beta$ or AICD. In contrast, accumulation of APP Cterminal stubs can occur in PSKO but not in APPKO/PS1KO cells establishing APP-CTFs as the causative APP fragments involved in Syt7 regulation.

We then performed experiments to better understand the molecular mechanism underlying the functional interaction between APP and Syt7. We tested whether the two proteins were expressed in synaptic compartments and whether they could physically interact. We detected both APP and Syt7 in synaptosomes prepared from adult mouse brain (Supplementary Fig. 7a) in agreement with previous studies ${ }^{10,12,29}$. Coimmunoprecipitation of the two proteins (in both directions) demonstrated that they are present in the same protein complexes (Fig. 7d). Additionally, we observed that the two proteins colocalize in neuronal cultures with a Manders coefficient of 0.77 (Supplementary Fig. 7b). Interestingly, colocalization increases (Manders coefficient of 0.94) following treatment of the neurons for 4 days with GSI suggesting that accumulated APP-CTFs also colocalize with Syt7 (Supplementary Fig. 7b).

We further investigated the relationship between APP-CTFs and Syt7 by performing cell fractionation on an iodixanol gradient. We observed that Syt7 was enriched in the lighter fractions from neuronal culture extracts characterized by high expression of VAMP2 (Fig. 7e). Interestingly, APP-CTFs was detected in the same fractions while full-length APP was 
a
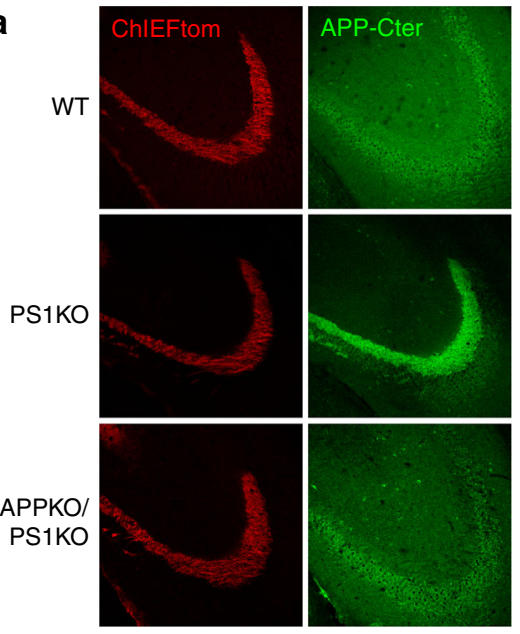

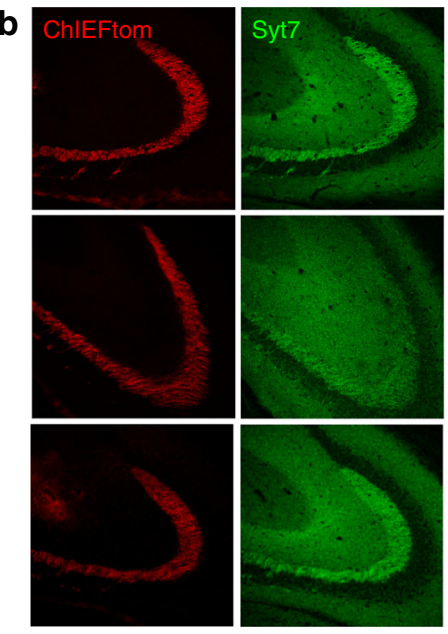

d

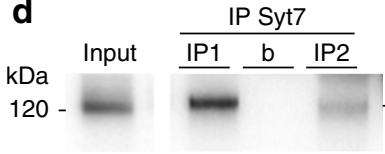

- APP-FL

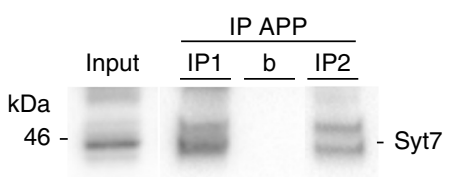

e

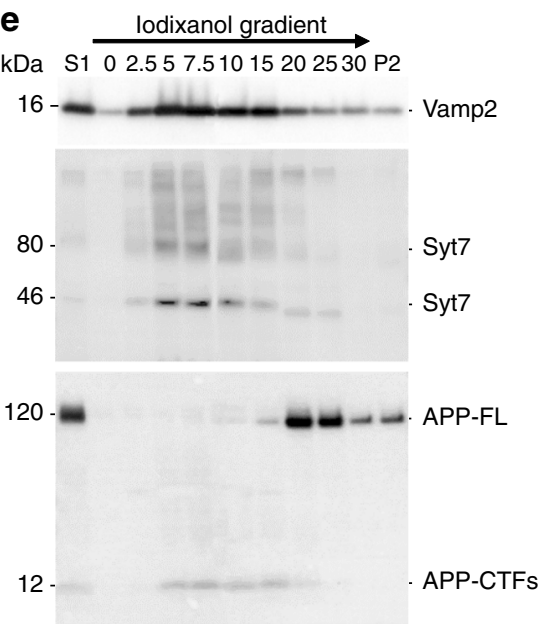

C

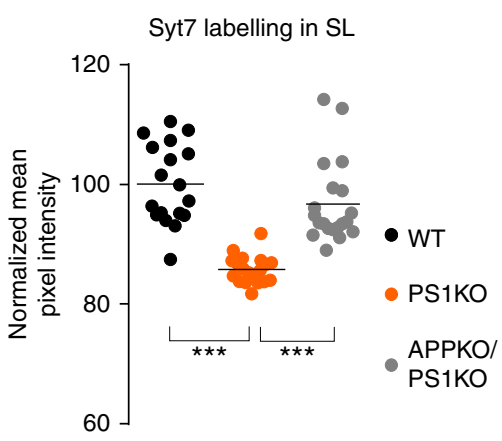

$\mathbf{f}$
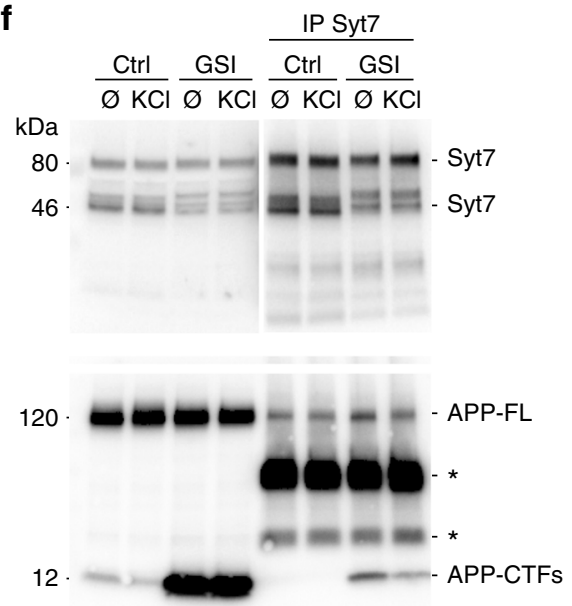

Fig. 7 PS regulation of Syt7 expression depends on APP-CTFs, new interacting partners of Syt7. a Confocal images of ChIEFtom (red channel) and APPCterminal (detected with C1/6.1 antibody, green channel) in the Mf tracts in WT, PS1KO, and PS1KO/APPKO conditions. Mfs are faintly labelled with the APP antibody in WT and barely in APPKO/PS1KO. In contrast, PS1KO Mfs display strong labelling of APP. b Confocal images as in a displaying Syt7 labelling. Mfs are strongly labelled in WT and in APPKO/PS1KO but faintly in PS1KO. c Scatter plots of the mean pixel intensity in the SL normalized to the mean pixel intensity in the stratum radiatum report a reduction of $18 \%$. In this condition, $50 \%$ of the GCs are infected by a Cre-expressing LV thus $~ 50 \%$ of the Mfs are projected from non-infected (PS expressing) GCs. d WB from synaptosomal samples prepared from whole brains of adult mice. Top: IP of Syt7 pulled-down APP-FL. Bottom: IP of APP pulled-down Syt7. The characterization of the synaptosomal fraction is depicted in Supplementary Fig. 7. b: beads + immuno-precipitating antibody without sample. e Western-blot of cellular fractions from cultured neurons separated on an iodixanol gradient. VAMP2, Syt7, and APP-CTFs are enriched in light fractions while APP-FL is present in heavier fractions. f Samples prepared from cultured neurons treated or not with GSI from 10 to $14 \mathrm{DIV}$ and with or without $\mathrm{KCI}(30 \mathrm{mM})$ for 15 min before lysis. In the inputs, detection of APP Cterminal with the Y188 antibody indicates that GSI induces CTFs accumulation. IP of Syt7 pulled-down APP-FL and CTFs. Asterisks indicate light and heavy chains of the immunoprecipitating antibody. Syt7/CTFs interaction is decreased by $\mathrm{KCl}$ treatment suggesting that it is regulated by neuronal activity

expressed in the more heavy fractions (Fig. 7e). This suggests a possible interaction between APP-CTFs and Syt7 in the lighter cellular compartments of the neurons. We thus studied a possible interaction between Syt7 and the APP fragments. Immunoprecipitation of Syt7 from protein lysates of neuronal cultures allowed co-precipitating APP indicating that the two proteins interact (Fig. 7f). When neurons were treated with GSI, APP-CTFs dramatically accumulated and was massively co-precipitated with Syt7 (Fig. 7f). Moreover, when the neuronal cultures were treated with $\mathrm{KCl}$ to depolarize the neurons and induce AP firing, the interaction between Syt7 and APP-CTFs decreased, indicating that the interaction was dynamically regulated by neuronal activity and possibly by SV release (Fig. $7 f$ ).

\section{Discussion}

Understanding the physiological function of $\mathrm{AD}$-related genes is fundamental because dysregulation of their normal function likely contributes to AD pathogenesis. Because APP and PS are abundantly expressed in presynaptic compartments ${ }^{10-12}$, where they interact with proteins of the release machinery, it is essential to understand their physiological role in presynaptic mechanisms. To address this, we have developed versatile optogenetic tools that help circumvent difficulties inherent to studies of presynaptic mechanisms with classical electrophysiological methods. Indeed, following genetic manipulations in a subpopulation of neurons, stimulation with an extracellular electrode activates both the axons from genetically manipulated cells and those from nonmodified cells. We cloned a new minimal promoter of the C1ql2 
gene and used it in a lentiviral vector to target expression of ChIEF in DG together with a second transgene, e.g. Cre recombinase. C1ql2-driven expression of ChIEF and Cre allowed the combination of region-specific activation with gene deletion in the DG of PS-conditional KO mice. Using this approach, we examined the effects of the genetic deletion of PS in presynaptic GCs on the morpho-functional properties of Mf-CA3 synapses.

We discovered that PS controls the levels of the $\mathrm{Ca}^{2+}$ sensor Syt7 presynaptically. Indeed, in the absence of presynaptic PS, Syt7 dramatically drops in axons and terminals. Interestingly, four other presynaptic markers tested were not impaired by the absence of PS, two being SV proteins (VAMP2 and Syt1) and two being active zone proteins (Bassoon and Syntaxin1A). Moreover, the absence of PS does not impact on the morphology of presynaptic terminals indicating, all together, that the functional phenotype is not caused by degenerative processes within presynaptic terminals.

The reduced expression of Syt7 in PSKO Mfs impairs two essential presynaptic functions: presynaptic facilitation and replenishment of release-competent vesicles. Indeed, Syt7 has recently been shown to be essential for presynaptic facilitation ${ }^{27}$ at several central synapses. Its role is especially noteworthy at MfCA3 synapses where the large presynaptic train facilitation is nearly abolished in Syt7 $\mathrm{KO}^{27}$. Thus, the impaired short-term plasticity observed at PSKO Mf-CA3 synapses is consistent with the decreased expression of Syt7 in Mf terminals. Additionally, Syt7 is necessary for the replenishment of release competent $\mathrm{SVs}^{28}$. We demonstrated that re-expression of Syt7 in PSKO MfCA3 synapses rescued presynaptic function, providing a causal link between the drop of Syt7 expression and the functional phenotype. Of note, quantal and basal transmission were unchanged in agreement with the observation that Syt7 does not determine the initial probability of release ${ }^{27,28}$.

Next, we investigated whether this novel synaptic function of PS depends on its $\gamma$-secretase proteolytic activity. In neuronal cultures, we observed that inhibiting $\gamma$-secretase activity pharmacologically decreased Syt7 expression within a few days indicating a role for $\gamma$-secretase. The investigation of molecular mechanisms involving $\gamma$-secretase is complex due to its diverse functions and molecular targets ${ }^{8}$. APP is a major substrate of PS and is highly enriched in synapses, and particularly at a presynaptic level ${ }^{12}$. In the absence of PS or when $\gamma$-secretase activity is inhibited, the substrates of PS, APP-CTFs, cannot be processed into $\mathrm{A} \beta$ (or $\mathrm{p} 3$ ) and AICD. Thus, in the PSKO condition, only the absence and not the excess of $A \beta$ (or p3) and AICD could potentially explain the PSKO phenotype. Because AICD has been proposed to act as a transcription factor ${ }^{31}$, the decline in Syt7 level could result from a deficit in AICD. However, the analysis of Syt7 mRNA and rescue experiments of AICD expression in PSKO GCs show that the absence of AICD does not play a role in the regulation of Syt7 expression and function.

We observed that the substrates of PS, APP-CTFs, accumulate in Mf axons in the absence of PS, or in cultured neurons when $\gamma$ secretase is pharmacologically inhibited. These fragments of APP are involved in the deficit in Syt7 expression observed in PSKO axons, since deletion of APP in APPKO/PS1KO prevents the drop of Syt7 expression. Moreover, there was an inverse relationship between APP-CTFs and Syt7 expression in neuronal cultures. Finally, we show that Syt7 is a new interacting partner of APP-CTFs. The mechanism linking the accumulation of APPCTFs to downregulation of Syt7 will need further investigation. A possible hypothesis is that CTFs sequester Syt7 in compartments preventing its functional roles and causing its degradation. This interpretation is supported by the well-documented function of PS and APP in axonal transport ${ }^{37-40}$ and by the observation that the absence of PS impairs the cell surface expression and the shuttling of several proteins between the cell interior and the cell surface $^{41,42}$. Syt7, as a protein expressed both in SVs and at the plasma membrane ${ }^{43,44}$ could be sequestered in the wrong compartment by accumulation of APP-CTFs. Our results are based on the pharmacological inhibition of $\gamma$-secretase activity or genetic invalidation of PS. They suggest that in physiological conditions, presynaptic $\gamma$-secretase activity regulates the abundance of Syt7, a key presynaptic protein involved in presynaptic plasticity which interacts with APP-CTFs. It will be interesting to investigate the physiological conditions under which $\gamma$-secretase activity is regulated, hence modulating the amount of APP-CTFs and consequently of Syt7.

Although our results mainly deal with the investigation of the physiological role of PS, they may also be of importance for the physiopathology of AD. FAD mutations of PS1 also cause APPCTFs accumulation due to decreased enzymatic activity of $\gamma$ secretase, limiting the catabolism of APP-CTFs ${ }^{45}$. Interestingly, genetic evidence indicates a relationship between the level of APP- $\beta C T F$ catabolized by $\gamma$-secretase and AD. Indeed, APP- $\beta$ CTF is highly produced when the APP gene contains mutations causing the early onset form of $\mathrm{AD}$ (the Swedish mutations $\mathrm{K} 595 \mathrm{~N} / \mathrm{M}^{2} 96 \mathrm{~L}^{2}$ ) and is produced to a much lower extent when APP contains a mutation (A598T) protecting from $\mathrm{AD}^{46}$. Overall our data highlight the notion that presynaptic dysfunction may play a role in AD in relation to APP processing by PS.

\section{Methods}

Ethical approval. All experiments were performed in accordance with the European Union (EU) guidelines (directive 2010/63/UE) and under the authorization of the 'Pole in Vivo' of the IINS (\#A33 12 061). The experimental protocol (\#50120174-A) has been authorized by the local ethical committee (registered at the National Comittee about Animal Experimentation).

Animals. The animals used in this study were PS1floxed \#007605, PS2-KO \#005617 mice obtained from Jackson Laboratory (Bar Harbor, ME, USA) and C57BL6/J wild type (WT). In the PS1floxed mice, loxP sites are present on either side of exon 7 of the targeted gene and allow deletion of this exon by Cre-mediated recombination. In the PS2 mouse line, a targeting vector was used to disrupt exon 5 and introduce a frame shift between exons 4 and 6 . We developed a PS1fl/PS2KO mouse line by crossing the PS1floxed and the PS2KO mice together. Mice were genotyped using the following primers: PS1fl-F: CAGGCACACTCACCCACAGA, PS1fl-R: GAAAATCATATCCCCTACACTA; PS2WT-F: GAGAGAAGGCACCA GGATAGGTT, PS2WT-R: AGTGTCCTGGAGCAGCAGATAC and PS2Mut-F: CGAAGGAGCAAAGCTGCTATTGG, PS2Mut-R: CTGGGTCTCCAGCAG TC TTC. We developed an APPfl/PS1KO mouse line by crossing the APPfloxed ${ }^{36}$ and the PS1floxed mice together. APP gene was genotyped using the following primers: 1: AACGCAGGGAGGAGTCAGGG, 2: TGCATGTCAGTCTAATGGAGGC, and 3: ATCTGCC CTTATCCAGTGAAATGAACC.

Cloning C1qI2 promoter and generation of lentiviral vectors. The lentiviruses were produced by the viral vectors facility of Bordeaux "TransBioMed". The lentiviruses developed here are based on the pRRLsin-PGK-MCS-WPRE plasmid where PGK promoter has been exchanged with the C1ql2 minimal promoter. This sequence was cloned from mouse genomic DNA using the following primers EcoRV/C1ql2-F: ATATATGATATCagcacccacatagcagc; BamH1/C1ql2-R: ATATATGGATCCgctctggaactgat ctg. The $1.3 \mathrm{~kb}$ sequence was then inserted between EcoRV and BamH1 restriction sites of the lentiviral backbone. After the promoter, the following cDNA sequences were inserted in the $5^{\prime}-3^{\prime}$ order: ChIEFtomato, $2 \mathrm{~A}$ peptide from porcine teschovirus- $1^{47}$, finally the Cre recombinase or the eGFP. A Cre-dependent construct was created to allow conditional expression of ChIEFtom and AICD. Behind C1q12 promoter, a Lox-Stop-Lox cassette was inserted followed by the cDNA of ChIEFtom, 2A peptide and AICD. A second Cre-ON construct was created based on a double-inverted open-reading frame (DIO) strategy. Briefly, a cassette including ChIEFtom, 2A peptide and Syt7 was placed in an inverted manner between two doublets of lox sequences (loxP and lox 2722). For 3D reconstruction of $\mathrm{Mf}$ boutons, a lentiviral vector expressing myristoylated YFP (mYFP) was used (generously provided by C. Lois, California Institute of Technology, Pasadena, CA) described in ref. ${ }^{26}$.

Viral gene transfer and stereotaxic delivery. Mice (P22-P30) were anesthetized by isoflurane inhalation and injected with buprenorphine to prevent post-surgery pain. $1 \mu \mathrm{L}$ of viral solution $\left(5 \times 10^{6}\right.$ particles $)$ was injected using a micropump and 
syringe (Nanofil WPI) at the rate of $100 \mathrm{nl} / \mathrm{min}$ in the DG (Y: $2.2 \mathrm{~mm}$ from lambda; $X: \pm 2.0 \mathrm{~mm}$ from sagittal suture; $Z: 2.0 \mathrm{~mm}$ from the skull) to infect GCs. This site targets a rather dorsal part of the hippocampus but the spreading of viruses allowed the infection of a large part of the hippocampus (radius of roughly $1 \mathrm{~mm}$ ) to produce numerous slices for IHC or electrophysiology. Experiments were performed at least 3 weeks post-injection.

Slice preparation. Mice were anesthetized using ketamine/xylasine mix $(100 \mathrm{mg} /$ $\mathrm{kg} / 10 \mathrm{mg} / \mathrm{kg}$; i.p.) and perfused transcardially with ice cold artificial cerebrospinal fluid (aCSF) for 40-60 s. The brain was quickly removed from the skull and chilled in ice-cold aCSF containing the following (in mM): $120 \mathrm{NaCl}, 26 \mathrm{NaHCO}_{3}, 2.5$ $\mathrm{KCl}, 1.25 \mathrm{NaH}_{2} \mathrm{PO}_{4}, 2 \mathrm{CaCl}_{2}, 1 \mathrm{MgCl}_{2}, 16.5$ glucose, 2.8 pyruvic acid, 0.5 ascorbic acid, $\mathrm{pH} 7.4$ adjusted by saturating with carbogen $\left(95 \% \mathrm{O}_{2}\right.$ and $\left.5 \% \mathrm{CO}_{2}\right)$, and an osmolarity of $305 \mathrm{mOsm}$. Parasagittal slices $(300 \mu \mathrm{m})$ were cut from brain hemispheres using a vibratome (VT 1200S, Leica Microsystems, Nussloch, Germany). Slices were then transferred to aCSF for $20 \mathrm{~min}$ at $33^{\circ} \mathrm{C}$ and thereafter maintained at room temperature until required.

Electrophysiological recordings. A slice was transferred to a recording chamber where it was submerged and continuously perfused with oxygenated $\left(95 \% \mathrm{O}_{2}, 5 \%\right.$ $\mathrm{CO}_{2}$ ) aCSF at RT. Whole-cell patch clamp recordings were performed on neurons identified with a differential interference contrast microscope (Eclipse FN-1, Nikon, Champigny sur Marne, France) equipped with a camera (CoolSNAP EZ, Ropper, Evry, France). Voltage-clamp recordings were performed on CA3 pyramidal neurons using patch electrode $(4 \pm 0.5 \mathrm{M} \Omega)$ filled with an internal solution containing the following (in $\mathrm{mM}$ ): $125 \mathrm{CsCH}_{3} \mathrm{SO}_{3}, 2 \mathrm{MgCl}_{2}, 4 \mathrm{NaCl}, 5$ phosphocreatine, $4 \mathrm{Na}_{2} \mathrm{ATP}, 10$ EGTA, 10 HEPES pH 7.3 adjusted with $\mathrm{CsOH}$, and an osmolarity of $300 \mathrm{mOsm}$. Bicuculline $(10 \mu \mathrm{M})$ was added to the bath to inhibit GABA-A receptors. Mf-CA3 excitatory post synaptic currents (EPSCs) were evoked by light pulses of $1 \mathrm{~ms}$ over GCs dendrites in the stratum moleculare. The baseline stimulation frequency for all experiments was $0.1 \mathrm{~Hz}$. Current-clamp recordings were performed on GCs or $\mathrm{CA} 3$ cells patched with an internal solution containing the following (in mM): $115 \mathrm{KC}_{6} \mathrm{H}_{11} \mathrm{O}_{7}, 10 \mathrm{KCl}, 0.02 \mathrm{CaCl}_{2}, 4 \mathrm{MgATP}$, $0.3 \mathrm{NaGTP}, 15$ phospho-creatine, $0.2 \mathrm{EGTA}, 10 \mathrm{HEPES} \mathrm{pH} 7.3$ adjusted with KOH and an osmolarity of $300 \mathrm{mOsm}$. Spike transmission experiments were performed in current clamp mode while using the "low-frequency voltage clamp" function of EPC10 amplifier to keep the membrane potential of the recorded CA3 neurons constant at $-70 \mathrm{mV}$ between recording sweeps. Signals were amplified using a HEKA EPC10 amplifier (Lambrecht, Germany), filtered at $3.3 \mathrm{kHz}$ and digitized at $10 \mathrm{kHz}$ via PatchMaster software (Lambrecht, Germany). Data were analyzed using IGOR PRO 6.3 (Wavemetrics, Lake Oswego, OR).

\section{Analysis of quantal transmission. The characteristics of quantal neuro-} transmission at Mf/CA3 were determined by recording EPSCs in CA3 neurons while stimulating with light-pulses at $1 \mathrm{~Hz}$ in presence of $32 \mu \mathrm{M}$ of cadmium. We used the following model to estimate the probability of release at individual releasing site (active zone) and we estimated the contamination of mono-quantal release by bi-quantal release in the experiments in presence of $32 \mu \mathrm{M}$ cadmium. In this condition, the failure rate is of $88 \%$, hence the probability of release is $12 \%$. This total probability is the sum of the probability of release at individual release sites (we considered 20 sites/Mf bouton here) and possibly of concomitant release from two sites (concomitant release from more than two sites are negligible and not considered here). For the simplicity of the demonstration, we postulate the release sites to be equivalent.

Thus, Eq. (1): $P($ tot $)=0.12=P(s 1)+P(s 2)+\ldots+P(s 20)+P(s 1+s 2)+$ $P(s 1+s 3)+\ldots+P(s 19+s 20)=20 P(s)+190 P(s+s)$

Eq. (2) of second order is: $190 P(s+s)+20 P(s)-0.12=0$ and its solutions are:

$P(s) 1=-0.11095$ and $P(s) 2=+0.00569$

Thus, Eq. (3): $P(s+s)=\left(0.12-20^{\star} 0.00569\right) / 190=0.0000316$. In our recordings, the ratio of release from two sites compared to one site is: $\left(190^{\star} 0.0000316\right) /\left(20^{*} 0.0057\right)=0.0526$, i.e. $5.26 \%$. In our condition $(32 \mu \mathrm{M}$ of cadmium) for the analysis of quantal release, $5 \%$ of the EPSCs were possibly coming for the concomitant release from two sites.

Immunostaining. WT and PSKO mice were anesthetized with intraperitoneal administration of pentobarbital ( $50 \mathrm{mg} / \mathrm{kg}$ body weight) and were perfused intracardiacally with $0.9 \% \mathrm{NaCl}$ for 1 min followed by $4 \%$ paraformaldehyde in $0.1 \mathrm{mM}$ PBS for 3 min for imaging experiments. Brain were removed and postfixed in $4 \%$ PFA for $8 \mathrm{~h}$. Coronal sections of the hippocampus $(50 \mu \mathrm{m})$ were cut on a vibratome (Leica VT1000S). Sections were permeabilized in $0.3 \%$ TritonX100 for $2 \mathrm{~h}$ at room temperature. Then sections were incubated with primary antibody diluted at $1 / 500$ in PBS-tween for $48 \mathrm{~h}$ at $4{ }^{\circ} \mathrm{C}$ (APP N-terminal JRD32 mouse antibody is a noncommercial antibody directed against the N-terminal APP-E1 domain ${ }^{48}$, APP Cterminal Y188 rabbit antibody Abcam \#32136 or APP C-terminal C1/6.1 mouse antibody BioLegend, BACE1 rabbit antibody D10E5 Cell Signaling \#5606; syntaxin1 rabbit antibody Alomone \# ANR-002; synaptotagmin7 mouse antibody MABN665, clone N275/14 Millipore; from Synaptic Systems: bassoon rabbit antibody \#141013, synaptoporin rabbit antibody \#102002, synaptotagmin1 mouse antibody \#105011, synaptotagmin7 rabbit antibody \#105173, synaptobrevin2 mouse antibody \#104211). After washes in PBS-T, sections were incubated with secondary antibodies for $3 \mathrm{~h}$ (coupled to either Alexa 488 or 555 or anti-rabbit Abberior STAR 635P). Slices were incubated during the penultimate wash in DAPI $(300 \mathrm{nM})$ for $10 \mathrm{~min}$ before a last PBS wash and mounted in Mowiol-based solution. For low-magnification acquisition, an up-right widefield microscope (Zeiss AxioPhot2) was used with a $\times 2.5$ objective. The camera was a CoolSnap HQ2 (Photometrics, Tucson, USA) controlled by MetaMorph.

Whole brain slice imaging. WT or PS1fl/PS2KO mice were injected with lentivirus encoding ChIEFtom2A-Cre on the right hemisphere and ChIEFtom2A-GFP on the left hemisphere using stereotaxic surgery. After fixation and brain slicing, whole brain sections, fluorescently labelled against synaptotagmin1, synaptotagmin7, or synaptobrevin2 were acquired using the digital slide scanner NanoZoomer 2.0HT (Hamamatsu).

Confocal fluorescence microscopy and image analysis. Confocal acquisition were performed using a Leica SP8 White Light Laser 2 on an inverted stand DMI6000 (Leica Microsystems, Mannheim, Germany) and with a ×20 (NA 0.75) objective. Images of focal planes in the region of the $S L$ were acquired in the red (ChIEFtomato) and far-red (synaptotagmin1, synaptotagmin7, synaptobrevin2, syntaxin1) channels. The settings (laser power, gain, offset) were fixed between genotypes. Images were analyzed using ImageJ. A region of interest (ROI) restricted to the Mf tract was defined in the red channel using the default mode threshold of ImageJ followed by the creation of a selection corresponding to this ROI. Then average pixel intensity was measured in the same ROI but in the far-red channel. The mean pixel intensity of a second area (ROI2) in the stratum radiatum (SR), an adjacent region not genetically manipulated was measured. A ROI/ROI2 ratio was performed slice per slice to normalize the data (Fig. 3b). Between 40 and 50 measurements from 6 to 8 mice were acquired per genotype and per presynaptic proteins studied.

Imaging of neuronal cultures and co-localization analysis. Confocal acquisition were performed using a Leica SP8 White Light Laser 2 on an inverted stand DMI6000 (Leica Microsystems, Mannheim, Germany) and with a 63× (NA 1.4) objective. Focal plane images were acquired for different cells in green (APP), red (Syt7), and far-red (MAP2) channels. The settings (laser power, gain, offset) were fixed between cells as well as the pixel size $(120 \mathrm{~nm})$. Images were analyzed by using Image J plugin JACoP ${ }^{49}$. Fluorescence was analyzed by excluding the cell body and applying a mean filter (radius $=1 \mathrm{px}$ ). For each image acquired the JACoP plugin was used to apply the Costes auto thresholding pixel intensity in each of the channels. Then, Manders coefficient was applied by the plugin to determine the amount of co-localized pixels in each channel, represented as 0 (no co-localization) or 1 (perfect co-localization)

Mf boutons analysis after 3D-stack acquisition. Confocal acquisition was performed using a Leica SP8 described above and with a $\times 63$ (NA 1.40) objectives. Images of the Mf boutons expressing myristoylated YFP in the CA3b-c region of hippocampus were captured with an interval of $0.2 \mu \mathrm{m}$ in $z$-axis and a pixel size of $70 \mu \mathrm{m} .200 \mathrm{Mf}$ boutons from five animals per genotype were analyzed. Highresolution 3D stacks were generated using Imaris 7.1.1 software (Bitplane) to characterize and analyze presynaptic terminals. Illustrations correspond to $3 \mathrm{D}$ reconstructions smoothened by a median filter.

STED microscopy and image analysis. STED acquisitions were performed on the same microscope using a $775 \mathrm{~nm}$ depletion laser and a $\times 100$ (NA 1.40) objective. Images of the Mf bouton were first acquired on the yellow channel (myristoylated YFP) to obtain a mask of the Mf bouton. An intensity threshold was applied to define the region contour of the Mf bouton. Then, a STED image of bassoon puncta was acquired in far-red with a pixel size of $20 \mathrm{~nm}$. To count the number of bassoon clusters per Mf bouton and to measure their surface, images were treated by a segmentation program allowing clusters to be detected as single objects, which were then automatically counted within the contour of Mf boutons. Analyses were performed with Image J software (National Institutes of Health, New York, USA).

Laser-assisted microdissection and RTqPCR. Laser capture microdissection (LCM) was performed using a MicroBeam microdissection system Version 4.8 equipped with a P.A.L.M. RoboSoftware (P.A.L.M. Microlaser Technologies AG, Bernried, Germany). Microdissection was performed at $\times 5$ magnification. Samples were collected in adhesives caps (P.A.L.M. Microlaser Technologies AG, Bernried, Germany).

cDNA were synthesized from total RNA using RevertAid Premium Reverse Transcriptase (Fermentas) and primed with oligo-dT primers (Fermentas) and random primers (Fermentas). qPCR was performed using a LightCycler ${ }^{\circledR} 480$ RealTime PCR System (Roche, Meylan, France). The PCR data were exported and analyzed with a software (Gene Expression Analysis Software Environment) developed at the NeuroCentre Magendie. For the determination of the reference gene, the Genorm method was used. Relative expression analysis was corrected for 
PCR efficiency and normalized against two reference genes: the peptidylprolyl isomerase A (Ppia) and non-POU domain containing, octamer-binding protein (Nono). The relative level of expression was calculated using the comparative $\left(2^{-\Delta \Delta C T}\right)$ method. Primers sequences are reported in supplementary Table 1.

Cell fractionation in iodixanol gradient. Neuronal cultures were homogenized in buffer A $(130 \mathrm{mM} \mathrm{KCl}, 25 \mathrm{mM} \mathrm{NaCl}, 25 \mathrm{mM}$ Tris- $\mathrm{HCl}$ pH 7.4, 1 mM EGTA, protease inhibitors) and centrifuged at $1000 \times g, 10 \mathrm{~min}$. The supernatant $\mathrm{S} 1$ was then transferred to gradient of iodixanol in buffer A and centrifuged at 122,000 $\times g$, $40 \mathrm{~min}$. Each fractions was then diluted in buffer A and centrifuged at $126,000 \times g$, $40 \mathrm{~min}$. Pellets were suspended in lysis buffer and analyzed by western-blotting.

Cell fractionation to prepare synaptosomes. Synapsomes were prepared from mouse brain following a published protocol ${ }^{50}$. Briefly, tissue samples were homogenized by 12 strokes at $900 \mathrm{rpm}$ with a glass/Teflon potter in $2 \mathrm{~mL}$ of ice cold isosmolar buffer ( $320 \mathrm{mM}$ sucrose, $4 \mathrm{mM}$ HEPES, pH 7.4). Then, the homogenate was centrifuged at $1000 \times g$ for $8 \mathrm{~min}$ at $4^{\circ} \mathrm{C}$. The supernatant $\mathrm{S} 1$ was then centrifuged at $12,500 \times \mathrm{g}$ for $13 \mathrm{~min}$ at $4{ }^{\circ} \mathrm{C}$. The pellet $\mathrm{P} 2$ was resuspended in isosmolar buffer and laid on top of a discontinuous sucrose gradient (composed of one phase at $0.8 \mathrm{M}$ sucrose, $4 \mathrm{mM}$ HEPES $\mathrm{pH} 7.4$ and one phase of $1.2 \mathrm{M}$ sucrose, $4 \mathrm{mM}$ HEPES $\mathrm{pH}$ 7.4). The sample was centrifuged at $50,000 \times \mathrm{g}$ for $1 \mathrm{~h} 10 \mathrm{~min}$ at $4^{\circ} \mathrm{C}$. The synaptosomal fraction at the interface of the two layers of sucrose was collected with a $1 \mathrm{~mL}$ syringe and a $25 \mathrm{G}$ needle by piercing the tube wall. The sample was resuspended in lysis buffer, compared to homogenate and subjected to immunoprecipitation.

Co-immunoprecipitation and western-blotting. Analysis of the expression of the different polypeptides expressed by lentiviral constructs was tested by transfecting HEK293 cells (from ATCC) with the non-liposomal reagent X-tremeGENE (Roche). Cells were scraped and lysed 2 days after transfection. Analysis of the expression level and the interaction of endogenous neuronal proteins were made from lysates of cortical neurons cultures for 9 DIV in Neurobasal (Gibco) and then up to 14DIV in BrainPhys. The $\gamma$-secretase inhibitor (L685,458) was applied from 9 DIV up to lysis. Cells were lysed in a dodecylmaltoside-based lysis-buffer (Hepes 50 mM, pH 7.4; NaCl 100 mM, Glycerol 10\%, DDM 0.5\%). Immunoprecipitation was performed using an antibody against Syt7 on 600-1000 $\mu$ g of cell lysates. Immunoglobulins-proteins complexes were pull down by addition of magnetic beads (surebeads) and magnets. Samples were completed with Laemmli buffer. Proteins in samples were separated by western-blotting using $4-20 \%$ gradient Tris-tricine gels. Membranes were probed for the proteins of interest and chemioluminescent bands detected with a CCD camera (ChemiDoc-BioRad). Full-length blots can be found in Supplementary Fig. 8 .

Statistical analysis and graphical representations. Statistical analyses were performed with Prism 6.0 (GraphPad Software, La Jolla, CA, USA). Values were first tested for normality (D'Agostino and Pearson omnibus test). Data were presented as scatter plots with mean or median according to the result of the normality test. Normally distributed data were compared using a $t$-test (two-sided) while Mann-Whitney rank test (two-sided) was used for non-normal data set. Analyses involving two factors (genotype, stimulus number) were presented as points with error bars $( \pm \mathrm{SEM})$ and non-linear fits. Statistical differences were tested using twoway ANOVAs. Cumulative frequency distributions were presented as fractions and tested using a Mann-Whitney rank test. Statistical differences were considered as significant at $p<0.05$.

Drugs. All drugs were obtained from Tocris biosciences or Sigma-Aldrich.

\section{Data availability}

All data are available from the authors upon request.

Received: 15 February 2018 Accepted: 19 September 2018

Published online: 14 November 2018

\section{References}

1. Sherrington, R. et al. Cloning of a gene bearing missense mutations in earlyonset familial Alzheimer's disease. Nature 375, 754-760 (1995).

2. Mullan, M. et al. A pathogenic mutation for probable Alzheimer's disease in the APP gene at the N-terminus of beta-amyloid. Nat. Genet. 1, 345-347 (1992).

3. De Strooper, B. et al. Deficiency of presenilin-1 inhibits the normal cleavage of amyloid precursor protein. Nature 391, 387-390 (1998).

4. Barthet, G., Georgakopoulos, A. \& Robakis, N. K. Cellular mechanisms of $\gamma$ secretase substrate selection, processing and toxicity. Prog. Neurobiol. 98, 166-175 (2012).
5. DeKosky, S. T. \& Scheff, S. W. Synapse loss in frontal cortex biopsies in Alzheimer's disease: correlation with cognitive severity. Ann. Neurol. 27, 457-464 (1990).

6. Hamos, J. E., DeGennaro, L. J. \& Drachman, D. A. Synaptic loss in Alzheimer's disease and other dementias. Neurology 39, 355-361 (1989).

7. Terry, R. D. et al. Physical basis of cognitive alterations in Alzheimer's disease: synapse loss is the major correlate of cognitive impairment. Ann. Neurol. 30, 572-580 (1991).

8. Ho, A. \& Shen, J. Presenilins in synaptic function and disease. Trends Mol. Med. 17, 617-624 (2011).

9. Müller, U. C., Deller, T. \& Korte, M. Not just amyloid: physiological functions of the amyloid precursor protein family. Nat. Rev. Neurosci. 18, 281-298 (2017).

10. Laßek, M. et al. Amyloid precursor proteins are constituents of the presynaptic active zone. J. Neurochem. 127, 48-56 (2013).

11. Lundgren, J. L. et al. ADAM10 and BACE1 are localized to synaptic vesicles. J. Neurochem. 135, 606-615 (2015).

12. Wilhelm, B. G. et al. Composition of isolated synaptic boutons reveals the amounts of vesicle trafficking proteins. Science 344, 1023-1028 (2014).

13. Jeon, A. H. W. et al. Interactome analyses of mature $\gamma$-secretase complexes reveal distinct molecular environments of presenilin (PS) paralogs and preferential binding of signal peptide peptidase to PS2. J. Biol. Chem. 288, 15352-15366 (2013).

14. Kohli, B. M. et al. Interactome of the amyloid precursor protein APP in brain reveals a protein network involved in synaptic vesicle turnover and a close association with Synaptotagmin-1. J. Proteome Res. 11, 4075-4090 (2012).

15. Kuzuya, A. et al. Identification of the novel activity-driven interaction between synaptotagmin 1 and presenilin 1 links calcium, synapse, and amyloid beta. BMC Biol. 14, 25 (2016).

16. Rebola, N., Carta, M. \& Mulle, C. Operation and plasticity of hippocampal CA3 circuits: implications for memory encoding. Nat. Rev. Neurosci. 18, 208-220 (2017).

17. Yan, X.-X. et al. Binding sites of gamma-secretase inhibitors in rodent brain: distribution, postnatal development, and effect of deafferentation. J. Neurosci. 24, 2942-2952 (2004).

18. Liu, F. et al. $\gamma$-secretase binding sites in aged and Alzheimer's disease human cerebrum: the choroid plexus as a putative origin of CSF A $\beta$. Eur. J. Neurosci. 37, 1714-1725 (2013)

19. Iijima, T., Miura, E., Watanabe, M. \& Yuzaki, M. Distinct expression of C1qlike family mRNAs in mouse brain and biochemical characterization of their encoded proteins. Eur. J. Neurosci. 31, 1606-1615 (2010).

20. Lin, J. Y., Lin, M. Z., Steinbach, P. \& Tsien, R. Y. Characterization of engineered channelrhodopsin variants with improved properties and kinetics. Biophys. J. 96, 1803-1814 (2009).

21. Bouhours, B., Gjoni, E., Kochubey, O. \& Schneggenburger, R. Synaptotagmin2 (Syt2) drives fast release redundantly with Syt1 at the output synapses of parvalbumin-expressing inhibitory neurons. J. Neurosci. 37, 4604-4617 (2017).

22. Marchal, C. \& Mulle, C. Postnatal maturation of mossy fibre excitatory transmission in mouse CA3 pyramidal cells: a potential role for kainate receptors. J. Physiol. 561, 27-37 (2004).

23. Sachidhanandam, S., Blanchet, C., Jeantet, Y., Cho, Y. H. \& Mulle, C. Kainate receptors act as conditional amplifiers of spike transmission at hippocampal mossy fiber synapses. J. Neurosci. 29, 5000-5008 (2009).

24. Rizzoli, S. O. \& Betz, W. J. Synaptic vesicle pools. Nat. Rev. Neurosci. 6, 57-69 (2005).

25. Schneggenburger, R., Meyer, A. C. \& Neher, E. Released fraction and total size of a pool of immediately available transmitter quanta at a calyx synapse. Neuron 23, 399-409 (1999).

26. Lanore, F. et al. Deficits in morphofunctional maturation of hippocampal mossy fiber synapses in a mouse model of intellectual disability. J. Neurosci. 32, 17882-17893 (2012).

27. Jackman, S. L., Turecek, J., Belinsky, J. E. \& Regehr, W. G. The calcium sensor synaptotagmin 7 is required for synaptic facilitation. Nature 529, 88-91 (2016).

28. Liu, H. et al. Synaptotagmin 7 functions as a $\mathrm{Ca} 2+$-sensor for synaptic vesicle replenishment. eLife 3, e01524 (2014).

29. Sugita, $\mathrm{S}$. et al. Synaptotagmin VII as a plasma membrane $\mathrm{Ca}(2+)$ sensor in exocytosis. Neuron 30, 459-473 (2001).

30. Fukuda, M., Ogata, Y., Saegusa, C., Kanno, E. \& Mikoshiba, K. Alternative splicing isoforms of synaptotagmin VII in the mouse, rat and human. Biochem. J. 365, 173-180 (2002).

31. Cao, X. \& Südhof, T. C. A transcriptionally [correction of transcriptively] active complex of APP with Fe65 and histone acetyltransferase Tip60. Science 293, 115-120 (2001).

32. Laird, F. M. et al. BACE1, a major determinant of selective vulnerability of the brain to amyloid-beta amyloidogenesis, is essential for cognitive, emotional, and synaptic functions. J. Neurosci. 25, 11693-11709 (2005). 
33. Beel, A. J. \& Sanders, C. R. Substrate specificity of gamma-secretase and other intramembrane proteases. Cell. Mol. Life Sci. 65, 1311-1334 (2008).

34. McCarthy, J. V., Twomey, C. \& Wujek, P. Presenilin-dependent regulated intramembrane proteolysis and gamma-secretase activity. Cell. Mol. Life Sci. 66, 1534-1555 (2009).

35. Barthet, G. et al. Inhibitors of $\gamma$-secretase stabilize the complex and differentially affect processing of amyloid precursor protein and other substrates. FASEB J. 25, 2937-2946 (2011).

36. Mallm, J.-P., Tschäpe, J.-A., Hick, M., Filippov, M. A. \& Müller, U. C. Generation of conditional null alleles for APP and APLP2. Genes. N. Y. N. 2000 48, 200-206 (2010).

37. Dolma, K. et al. Presenilin influences glycogen synthase kinase-3 $\beta$ (GSK-3 $\beta$ ) for kinesin-1 and dynein function during axonal transport. Hum. Mol. Genet. 23, 1121-1133 (2014).

38. Kamal, A., Almenar-Queralt, A., LeBlanc, J. F., Roberts, E. A. \& Goldstein, L. S. Kinesin-mediated axonal transport of a membrane compartment containing beta-secretase and presenilin-1 requires APP. Nature 414, 643-648 (2001).

39. Pigino, G. et al. Alzheimer's presenilin 1 mutations impair kinesin-based axonal transport. J. Neurosci. 23, 4499-4508 (2003).

40. $\mathrm{Xu}, \mathrm{W}$. et al. Amyloid precursor protein-mediated endocytic pathway disruption induces axonal dysfunction and neurodegeneration. J. Clin. Invest. 126, 1815-1833 (2016).

41. Barthet, G. et al. Presenilin mediates neuroprotective functions of ephrinB and brain-derived neurotrophic factor and regulates ligand-induced internalization and metabolism of $\mathrm{EphB} 2$ and TrkB receptors. Neurobiol. Aging 34, 499-510 (2013).

42. Tamboli, I. Y. et al. Loss of gamma-secretase function impairs endocytosis of lipoprotein particles and membrane cholesterol homeostasis. J. Neurosci. 28, 12097-12106 (2008).

43. Dean, C. et al. Axonal and dendritic synaptotagmin isoforms revealed by a pHluorin-syt functional screen. Mol. Biol. Cell 23, 1715-1727 (2012).

44. Li, Y. C., Chanaday, N. L., Xu, W. \& Kavalali, E. T. Synaptotagmin-1- and synaptotagmin-7-dependent fusion mechanisms target synaptic vesicles to kinetically distinct endocytic pathways. Neuron 93, 616-631 (2017). e3.

45. Li, N. et al. Effect of presenilin mutations on APP cleavage; insights into the pathogenesis of FAD. Front. Aging Neurosci. 8, 51 (2016).

46. Jonsson, T. et al. A mutation in APP protects against Alzheimer's disease and age-related cognitive decline. Nature 488, 96-99 (2012).

47. Szymczak-Workman, A. L., Vignali, K. M. \& Vignali, D. A. A. Design and construction of 2A peptide-linked multicistronic vectors. Cold Spring Harb. Protoc. 2012, 199-204 (2012).

48. Hick, M. et al. Acute function of secreted amyloid precursor protein fragment APPsa in synaptic plasticity. Acta Neuropathol. 129, 21-37 (2015).

49. Bolte, S. \& Cordelières, F. P. A guided tour into subcellular colocalization analysis in light microscopy. J. Microsc. 224, 213-232 (2006).

50. Luquet, E., Biesemann, C., Munier, A. \& Herzog, E. Purification of synaptosome populations using fluorescence-activated synaptosome sorting. Methods Mol. Biol. 1538, 121-134 (2017).

\section{Acknowledgements}

We thank John Lin from UCSD-HHMI for his generous gift of ChIEFtdTomato plasmids. Lentiviruses were produced at the TransBioMed facility of the Bordeaux University. Cellular imaging was performed at the Bordeaux Imaging Center, a service unit of the CNRS-INSERM and Bordeaux University, member of the national infrastructure France BioImaging. Microdissections assisted by laser capture and RT-qPCR were performed at the facilities of the Neurocentre Magendie. Gael Barthet is supported by a young researcher position from the Fondation Plan Alzheimer and by a return-post-doc fellowship from the French National Agency for Research (ANR). This project has received funding from the European Union's Horizon 2020 research and innovation programme under grant agreement No. 676144. U.M. is supported by a grant from the Deutsche Forschungsgemeinschaft (MU 1457/14-1). We are grateful to Christophe Blanchet, Thierry Amédée, and Ruth Betterton for their careful comments on the manuscript.

\section{Author contributions}

G.B., T.J.S., J.R-.M. and F.B. conducted the experiments, G.B., U.M., and C.M. designed the experiments and wrote the manuscript.

\section{Additional information}

Supplementary Information accompanies this paper at https://doi.org/10.1038/s41467018-06813-x.

Competing interests: The authors declare no competing interests.

Reprints and permission information is available online at http://npg.nature.com/ reprintsandpermissions/

Publisher's note: Springer Nature remains neutral with regard to jurisdictional claims in published maps and institutional affiliations.

(c) Open Access This article is licensed under a Creative Commons Attribution 4.0 International License, which permits use, sharing adaptation, distribution and reproduction in any medium or format, as long as you give appropriate credit to the original author(s) and the source, provide a link to the Creative Commons license, and indicate if changes were made. The images or other third party material in this article are included in the article's Creative Commons license, unless indicated otherwise in a credit line to the material. If material is not included in the article's Creative Commons license and your intended use is not permitted by statutory regulation or exceeds the permitted use, you will need to obtain permission directly from the copyright holder. To view a copy of this license, visit http://creativecommons.org/ licenses/by/4.0/.

(C) The Author(s) 2018 